\title{
Characterization and antidiabetic activity of salicylhydrazone Schiff base vanadium(IV) and (V) complexes
}

\author{
Janusz Szklarzewicz ${ }^{1}$ • · Anna Jurowska ${ }^{1} \cdot$ Maciej Hodorowicz ${ }^{1} \cdot$ Grzegorz Kazek $^{2}$ - Barbara Mordyl $\left.\right|^{2}$. \\ Elżbieta Menaszek ${ }^{2} \cdot$ Jacek Sapa ${ }^{2}$
}

Received: 28 September 2020 / Accepted: 31 October 2020 / Published online: 24 December 2020

(c) The Author(s) 2020

\begin{abstract}
Twenty-four oxidovanadium(IV,V) complexes with tridentate Schiff base ligands based on 5-nitrosalicylaldehyde, 5-methoxysalicylaldehyde, or 5-sulfosalicylaldehyde and respective hydrazide were isolated, and characterized using physicochemical and spectroscopic methods. Three of them were structurally characterized by single-crystal X-ray structure determination. The biological activity studies included inhibition of human tyrosine phosphatases, studies on myocyte $\mathrm{C} 2 \mathrm{C} 12$, adipocyte 3T3-L1, and human hepatocyte HepG2 cell lines, glucose uptake in myocytes and adipocytes, and cytotoxicity tests. The complexes that were unstable in solutions showed biological activity typical of other V(IV) complexes, while the stable one showed much higher, ligand-dependent, activity.
\end{abstract}

\section{Introduction}

Vanadium compounds are of great importance not only in science but also in industry, including the very popular $\mathrm{V}_{2} \mathrm{O}_{5}$ used as a catalyst [1-3]. Its variety of oxidation states (typically ranging from II to $\mathrm{V}$ ), coordination numbers, and biological activity result in frequent use of this metal in coordination chemistry, especially because vanadium in different oxidation states forms a range of stable compounds, making synthesis relatively simple. In the course of our investigation connected with the insulin-mimetic properties of vanadium compounds, it is worth noting that both inorganic complexes (including $\mathrm{V}_{2} \mathrm{O}_{5}$ and $\mathrm{VOSO}_{4}$ ) and those with organic ligands are found to be active. Inorganic compounds were studied first, but it was soon shown (by McNeill) that organic compounds of vanadium work much better, and today bis(maltolato)oxidovanadium(IV) (BMOV) is used as

Electronic supplementary material The online version of this article (https://doi.org/10.1007/s11243-020-00437-1) contains supplementary material, which is available to authorized users.

Janusz Szklarzewicz

szklarze@chemia.uj.edu.pl

1 Faculty of Chemistry, Jagiellonian University, Gronostajowa 2, 30-387 Kraków, Poland

2 Faculty of Pharmacy, Jagiellonian University Medical College, Medyczna 9, 30-001 Kraków, Poland a standard for investigation of insulin-mimetic activity [4-8]. Soon, other biological activities were recognized—such as anticancer, antifungal or antibacterial, including activity toward protection from anticancer chemotherapy resistance [9-14]. Depending on the type of vanadium complex and ligands used, vanadium compounds exhibit large differences in terms of their structure and properties, and thus show different permeability through the cell membrane.

The best known and most widely described mechanism of the insulin-mimetic action of vanadium and its complexes in activation of cellular glucose uptake is inhibition of protein tyrosine phosphatases, especially PTP1B (but also TCPTP, PTP-MEG2, SHP-1, SHP-2, and LAR) [15-17]. Phosphatases are a group of enzymes that hydrolyze monoesters of phosphoric acid to a phosphate group and molecules with a free hydroxyl group. By removing a phosphate group, protein phosphatases can modulate the activity of cellular proteins and regulate cell metabolism by affecting many signal transduction pathways. Protein tyrosine phosphatases (PTPs) play an important role in the pathomechanisms of numerous diseases, thus many of them are potential pharmacological targets. In the glucose uptake process, this leads to maintenance of the phosphorylated state of the insulin receptor and thus to upstreaming signaling, which is similar to the action of the insulin hormone [18]. However, the list of molecular targets identified for vanadium action is much wider. Other enzymes (for example, kinases) in the glucose uptake pathway are also important, but the insulin-like effects of 
vanadium have not yet been fully clarified [19]. All this leads to modulation of various cellular functions and may be a basis for understanding the therapeutic role of vanadium compounds in metabolic, neoplastic, inflammatory, neurodegenerative, and other diseases.

We present herein the synthesis and physicochemical characterization of vanadium $(\mathrm{IV}, \mathrm{V})$ complex with tridentate ONO hydrazone Schiff base ligands, now extended to those based on 5-nitrosalicylaldehyde, 5-methoxysalicylaldehyde, and 5-sulfosalicylaldehyde with a set of hydrazides used by us previously [20-28]. As shown earlier, for tridentate contrary to bidentate ligands, a huge change of biological activity is observed, indicating that complexes in unchanged form (or at least with ONO ligand still coordinated to vanadium) pass into cells. Single-crystal X-ray structure analysis of three complexes as well as elemental analysis, magnetic susceptibility measurements, infrared (IR) and ultraviolet-visible (UV-Vis) spectroscopies, thermogravimetric measurements, and cyclic voltammetry are presented and discussed. To increase the stability of the complexes and fill the coordination sphere of V(IV/V), 2,2'-bipyridine (bpy) or 1,10-phenanthroline (phen) was used as co-ligand.

The importance of the PTPs tested in this study (PTP1B, SHP1, SHP2, and LAR) in metabolic disorders and diabetes has been discussed in literature [29,30]. Due to the low specificity of vanadium compounds in inhibiting the activity of individual PTPs and the potential adverse effects associated with this, the ability to inhibit CD45 phosphatase was examined. This phosphatase is associated with a possible impairment of hematopoietic cells and immune system disturbances [31].

\section{Experimental}

\section{Materials and methods}

[VO(acac $\left.)_{2}\right], \mathrm{VOSO}_{4} \mathrm{aq}, 1,10$-phenanthroline (phen), 2,2'-bipyridine (bpy), 5-nitrosalicylaldehyde, 5-methoxysalicylaldehyde, 5-sulfosalicylaldehyde, benzhydrazide, nicotinic hydrazide, phenylacetic acid hydrazide, 4-chlorobenzhydrazide, 2-hydroxybenzhydrazide, 4-hydroxybenzhydrazide, 4-methoxybenzhydrazide, and 3-hydroxy2-naphthoic acid hydrazide were of analytical grade (Aldrich or Alfa Aesar) and used as supplied. bis(maltolato) oxovanadium(IV) (BMOV) was synthesized according to literature method [8]. Ethanol (98\%) of pharmaceutical grade was from Polmos and used as supplied. All other solvents were of analytical grade (Aldrich) and used as supplied. $\mathrm{BaSO}_{4}$ was of spectroscopic grade (Japan). $\mathrm{Bu}_{4} \mathrm{NPF}_{6}$ was synthesized from $\mathrm{Bu}_{4} \mathrm{NBr}$ and $\mathrm{KPF}_{6}$ by a standard method and recrystallized from acetone [32]. Microanalysis of carbon, hydrogen, nitrogen, and sulfur was performed using an Elementar Vario MICRO Cube elemental analyzer. IR spectra were recorded on a Bruker EQUINOX 55 FT-IR spectrophotometer in $\mathrm{KBr}$ pellets. Electronic absorption spectra were recorded with a Shimadzu UV-3600 UV-Vis-NIR spectrophotometer equipped with a CPS-240 temperature controller. Diffuse reflectance spectra were measured in $\mathrm{BaSO}_{4}$ pellets with $\mathrm{BaSO}_{4}$ as reference on a Shimadzu 2101PC equipped with an ISR-260 integratingsphere attachment. Magnetic susceptibility measurements were performed on a Sherwood Scientific magnetic susceptibility balance. Cyclic voltammetry measurements were carried out in dimethyl sulfoxide (DMSO) with $\left[\mathrm{Bu}_{4} \mathrm{~N}\right] \mathrm{PF}_{6}$ $(0.1 \mathrm{M})$ as supporting electrolyte, using $\mathrm{Pt}$ working and counter and $\mathrm{Ag} / \mathrm{AgCl}$ reference electrodes on an Autolab PGSTAT128N potentiostat/galvanostat. $E_{1 / 2}$ values were calculated from the average anodic and cathodic peak potentials as $E_{1 / 2}=0.5\left(E_{\mathrm{a}}+E_{\mathrm{c}}\right)$. Redox potentials were calibrated versus ferrocene $(0.440 \mathrm{~V}$ versus standard hydrogen electrode (SHE)), which was used as an internal potential standard for measurements in organic solvents to avoid the influence of a liquid junction potential; the final values are reported versus SHE. Thermogravimetric measurements were performed on a Mettler Toledo TGA/SDTA 851e microthermogavimeter at scan speed of $10^{\circ} / \mathrm{min}$ in the $25-755^{\circ} \mathrm{C}$ range in air.

\section{Inhibition of human tyrosine phosphatases}

For this determination, the ability of the tested compounds to inhibit tyrosine phosphatases human recombinant protein was used. To solution of the tested complexes in black 384-well microplate wells (PerkinElmer), an equal volume of test solution of phosphatase was added in reaction buffer [25 mM 3-( $N$-morpholino) propanesulfonic acid (MOPS), $50 \mathrm{mM} \mathrm{NaCl}, 1 \mathrm{mM}$ dithiothreitol (DTT), and $0.05 \%$ Tween$20, \mathrm{pH}$ 7.0]. The final concentration of each of phosphatase was as follows: $50 \mathrm{ng} / \mathrm{ml}$, SHP1 $400 \mathrm{ng} / \mathrm{ml}$, SHP2 $50 \mathrm{ng} / \mathrm{ml}$, LAR $5 \mathrm{ng} / \mathrm{ml}$, and CD45 $100 \mathrm{ng} / \mathrm{ml}$. After $10 \mathrm{~min}$, solution of phosphate 6,8-difluoro-4-methyl (DiFMUP) was added to final concentration of $0.1 \mathrm{mM}$. After $20 \mathrm{~min}$ of incubation at room temperature, the fluorescence intensity (excitation at 355 and emission at $560 \mathrm{~nm}$ ) was measured on a multifunctional PTP1B POLARstar Omega plate reader (BMG Labtech, Germany). Assays were performed in triplicate. Results are expressed as the percent of inhibition of untreated control (enzyme with solvent only) [33].

\section{Cell models}

Myocyte C2C12 cell line (ATCC CRL-1772), a subclone of myoblasts from mouse muscles, was cultured according to standard protocol in Dulbecco's modified Eagle's medium (DMEM) supplemented with $10 \%$ fetal calf serum, $100 \mathrm{IU} /$ $\mathrm{ml}$ penicillin, and $100 \mu \mathrm{g} / \mathrm{ml}$ streptomycin at $37{ }^{\circ} \mathrm{C}$ in $5 \%$ 
$\mathrm{CO}_{2}$. Cells were plated on 96-well microplate and after reaching confluence differentiated in medium with $2 \%$ horse serum. Differentiation medium was changed every $72 \mathrm{~h}$.

Adipocyte 3T3-L1 cell line (ATCC CRL-11605), derived from fibroblasts from mouse embryo tissue, was cultured according to standard protocol in DMEM medium supplemented with $10 \%$ bovine calf serum, $100 \mathrm{IU} / \mathrm{ml}$ penicillin, and $100 \mu \mathrm{g} / \mathrm{ml}$ streptomycin at $37^{\circ} \mathrm{C}$ in $5 \% \mathrm{CO}_{2}$. Cells were seeded in 96-well poly-D-lysine-coated plates and cultured to reach confluence. The medium was then switched to differentiation medium and changed every $48 \mathrm{~h}$.

Human hepatocyte HepG2 cell line (ATCC HB-8065) was cultured according to standard protocol in DMEM supplemented with $10 \%$ fetal bovine serum, $100 \mathrm{IU} / \mathrm{ml}$ penicillin, and $100 \mu \mathrm{g} / \mathrm{ml}$ streptomycin at $37{ }^{\circ} \mathrm{C}$ in $5 \% \mathrm{CO}_{2}$. Cells were plated on 96-well microplate.

\section{Glucose uptake in myocytes and adipocytes}

Myocytes $\mathrm{C} 2 \mathrm{C} 12$ after 8-day differentiation and adipocytes 3T3-L after 11 days of differentiation were cultured and maintained as described above but plated on 96-well microplate coated with solid-phase scintillator (ScintiPlate, PerkinElmer, USA).

Myocytes were washed, and medium was changed for medium with $0.5 \%$ bovine serum albumin (BSA) instead of serum. After $24 \mathrm{~h}$ of incubation, tested complexes were added at final concentration of $50 \mu \mathrm{M}$. After $2 \mathrm{~h}$ of incubation at $37{ }^{\circ} \mathrm{C}$ in $5 \% \mathrm{CO}_{2}$, medium was changed for lowglucose $(1000 \mathrm{mg} / \mathrm{l})$ medium, tested complexes were added again, and further incubation for $4 \mathrm{~h}$ was conducted. Next, cells were washed three times with Krebs-Ringer buffer (KRB) without glucose ( $1 \mathrm{mM} \mathrm{MgSO}_{4}, 1 \mathrm{mM} \mathrm{CaCl}$, $136 \mathrm{mM} \mathrm{NaCl}, 4.7 \mathrm{mM} \mathrm{KCl}$, and $10 \mathrm{mM}$ HEPES; $\mathrm{pH}$ 7.4) and KRB with human recombinant insulin at final concentration $100 \mathrm{nM}$ (Sigma-Aldrich) was added for $15 \mathrm{~min}$. Wortmannin at final concentration of $200 \mathrm{nM}$ (Sigma-Aldrich) was used as negative control. After that, cells were washed three times with cold KRB and cytochalasin B (10 $\mu \mathrm{M}$ final) was added to several wells as "no uptake" control, proceeding as described below.

Adipocytes were washed, and medium was changed for basal medium. After $24 \mathrm{~h}$ of incubation, tested complexes were added at final concentration of $50 \mu \mathrm{M}$. After $2 \mathrm{~h}$ of incubation at $37{ }^{\circ} \mathrm{C}$ in $5 \% \mathrm{CO}_{2}$, medium was changed for DMEM medium without glucose, with $0.1 \%$ BSA free from fatty acid, $200 \mathrm{mM}$ L-glutamine, and $100 \mathrm{mM}$ pyruvate. Tested complexes were added again, and further incubation for $4 \mathrm{~h}$ was conducted. Next, cells were washed three times with KRB without glucose, and KRB with human recombinant insulin at final concentration of $100 \mathrm{nM}$ (SigmaAldrich) was added for $15 \mathrm{~min}$. Wortmannin at final concentration of $200 \mathrm{nM}$ (Sigma-Aldrich) was used as negative control. After that, cells were washed three times with cold $\mathrm{KRB}$, and cytochalasin B (10 $\mu \mathrm{M}$ final) was added for several wells as "no uptake."

For myocytes and adipocytes processed as described above, 2-deoxy-D-[U-14C]-glucose solution in KRB with total activity of $0.03 \mu \mathrm{Ci}$ was added to each well. After $1 \mathrm{~h}$ of incubation at $37{ }^{\circ} \mathrm{C}$ and $5 \% \mathrm{CO}_{2}$, uptake was blocked by adding cytochalasin B (10 $\mu \mathrm{M}$ final). Radioactivity of samples was measured using a MicroBeta Trilux 1450 scintillation counter (PerkinElmer, USA). Nonspecific radioactivity was subtracted from each result (cpm). Two independent experiments in triplicate were conducted. Final results are expressed as percent of control containing solvent only instead of tested compound.

\section{Viability and cytotoxicity tests}

After differentiation of C2C12 myocytes, medium was changed for medium with $0.2 \%$ bovine serum albumin, $100 \mathrm{IU} / \mathrm{ml}$ penicillin, and $100 \mu \mathrm{g} / \mathrm{ml}$ streptomycin, and after $2 \mathrm{~h}$ of incubation medium was changed for fresh medium containing vanadium complexes. After $48 \mathrm{~h}$ of incubation of 3T3-L1 adipocytes, medium was changed for standard medium with human recombinant insulin and tested vanadium complexes. Hepatocytes HepG2 were used for experiments after seeding for 18-24 h and after medium exchange. Vanadium complexes were tested at final concentration of $50 \mu \mathrm{M}$. After $24 \mathrm{~h}$ of incubation with tested compounds at $37{ }^{\circ} \mathrm{C}$ in $5 \% \mathrm{CO}_{2}, 10 \mu \mathrm{l}$ PrestoBlue cell viability reagent (Thermo Fisher Scientific) was added to each microplate well, and the microplate was placed on an orbital shaker for content mixing. Plates were incubated for $20 \mathrm{~min}$, and the fluorescence intensity at $560 \mathrm{~nm}$ excitation and $590 \mathrm{~nm}$ emission was determined using a POLARstar Omega multimode microplate reader (BMG Labtech, Germany). The results were normalized to untreated control (cells with solvent only), whose fluorescence intensity was taken as $100 \%$. Incubations of vanadium complexes with cells were performed in triplicate, and each compound was tested in three to four independent experiments. Similarly, cytotoxicity tests based on assessment of integrity of HepG2 hepatocyte membrane were performed using ToxiLight cytotoxicity kit (Lonza) according to manufacturer protocol.

\section{Synthesis}

Table 1 presents the Schiff base ligand notation and ligand components.

\section{Preparation of $\left[\left\{\mathrm{VO}\left(\mathrm{HL}_{1}\right)\left(\mathrm{H}_{2} \mathrm{O}\right)_{2}\right\}_{2}\left(\mathrm{SO}_{4}\right)\right] \cdot 1.5 \mathrm{H}_{2} \mathrm{O}(1)$}

5-Nitrosalicylaldehyde (500 mg, $3 \mathrm{mmol}$ ) and benzhydrazide (410 mg, $3 \mathrm{mmol}$ ) in $25 \mathrm{ml} \mathrm{EtOH}$ were refluxed for $10 \mathrm{~min}$ 
Table 1 List of ligands and ligand components

\begin{tabular}{|c|c|c|}
\hline Ligand notation & Aldehyde & Hydrazide \\
\hline $\mathrm{H}_{2} \mathrm{~L}_{1}$ & 5-Nitrosalicylaldehyde & Benzhydrazide \\
\hline $\mathrm{H}_{2} \mathrm{~L}_{2}$ & 5-Nitrosalicylaldehyde & 2-Hydroxybenzhydrazide \\
\hline $\mathrm{H}_{2} \mathrm{~L}_{3}$ & 5-Nitrosalicylaldehyde & 4-Hydroxybenzhydrazide \\
\hline $\mathrm{H}_{2} \mathrm{~L}_{4}$ & 5-Nitrosalicylaldehyde & Phenylacetic acid hydrazide \\
\hline $\mathrm{H}_{2} \mathrm{~L}_{5}$ & 5-Nitrosalicylaldehyde & Nicotinic hydrazide \\
\hline $\mathrm{H}_{2} \mathrm{~L}_{6}$ & 5-Nitrosalicylaldehyde & $\begin{array}{l}\text { 3-Hydroxy-2-naphthoic acid } \\
\text { hydrazide }\end{array}$ \\
\hline $\mathrm{H}_{2} \mathrm{~L}_{7}$ & 5-Methoxysalicylaldehyde & Benzhydrazide \\
\hline $\mathrm{H}_{2} \mathrm{~L}_{8}$ & 5-Methoxysalicylaldehyde & 2-Hydroxybenzhydrazide \\
\hline $\mathrm{H}_{2} \mathrm{~L}_{9}$ & 5-Methoxysalicylaldehyde & 4-Hydroxybenzhydrazide \\
\hline $\mathrm{H}_{2} \mathrm{~L}_{10}$ & 5-Methoxysalicylaldehyde & 4-Chlorobenzhydrazide \\
\hline $\mathrm{H}_{2} \mathrm{~L}_{11}$ & 5-Methoxysalicylaldehyde & Phenylacetic acid hydrazide \\
\hline $\mathrm{H}_{2} \mathrm{~L}_{12}$ & 5-Methoxysalicylaldehyde & Nicotinic hydrazide \\
\hline $\mathrm{H}_{2} \mathrm{~L}_{13}$ & 5-Methoxysalicylaldehyde & $\begin{array}{l}\text { 3-Hydroxy-2-naphthoic acid } \\
\text { hydrazide }\end{array}$ \\
\hline $\mathrm{H}_{2} \mathrm{~L}_{14}$ & 5-SO ${ }_{3}$-salicylaldehyde & 4-Hydroxybenzhydrazide \\
\hline $\mathrm{H}_{2} \mathrm{~L}_{15}$ & 5-SO ${ }_{3}$-salicylaldehyde & 4-Methoxybenzhydrazide \\
\hline $\mathrm{H}_{2} \mathrm{~L}_{16}$ & 5-SO ${ }_{3}$-salicylaldehyde & Phenylacetic acid hydrazide \\
\hline $\mathrm{H}_{3} \mathrm{~L}_{17}$ & 5-SO ${ }_{3}$-salicylaldehyde & Nicotinic hydrazide \\
\hline $\mathrm{H}_{3} \mathrm{~L}_{18}$ & 5-SO ${ }_{3}$-salicylaldehyde & $\begin{array}{l}\text { 3-Hydroxy-2-naphthoic acid } \\
\text { hydrazide }\end{array}$ \\
\hline
\end{tabular}

under Ar. Then, $\mathrm{VOSO}_{4}$ (657 mg, $3 \mathrm{mmol}$ ) was added, and the mixture was refluxed for $40 \mathrm{~min}$. At this stage, mixture was filtered off and the residue removed. The filtrate was concentrated to $50 \%$ of initial volume and cooled, and formed crystals were filtered off. Complex was washed three times with ethanol and dried in air. Yield $0.554 \mathrm{~g}$, $42 \% \mathrm{MW}=897.50$. Anal. Calc. for $\mathrm{C}_{28} \mathrm{H}_{31} \mathrm{~N}_{6} \mathrm{O}_{19.5} \mathrm{SV}_{2}: \mathrm{C}$, 37.47; H, 3.48; N, 9.36; S, 3.61\%. Found: C, 37.83; H, 3.55; $\mathrm{N}, 8.94 ; \mathrm{S} \sim 4 \%$. The complex is paramagnetic $\mu_{\mathrm{eff}}=1.80 \mu_{\mathrm{B}}$. FT-IR (KBr, cm ${ }^{-1}$ ): 3405 (w), 3299 (w), 3193 (w), 3075 (w), 1606 (vs), 1558 (s), 1489 (w), 1388 (m), 1337 (vs), 1317 (vs), 1248 (w), 1192 (w), 1131 (w), 1095 (m), 1041 (w), 959 (m), 904 (w), $837(\mathrm{w}), 808$ (w), 751 (w), 705 (m), 659 (w), 569 (w), 514 (w), 475 (w), 451 (w). UV-Vis (DMSO): 324, $390 \mathrm{~nm},(\mathrm{MeCN}): 227,290 \mathrm{~nm},(\mathrm{EtOH}): 317,371 \mathrm{~nm}$.

\section{Preparation of $\left[\mathrm{VO}\left(\mathrm{L}_{1}\right)(\mathrm{bpy})\right] \cdot 0.5 \mathrm{EtOH} \cdot 0.5 \mathrm{H}_{2} \mathrm{O}(2)$}

5-Nitrosalicylaldehyde (249 $\mathrm{mg}, 1.5 \mathrm{mmol}$ ) and benzhydrazide (204 mg, $1.5 \mathrm{mmol}$ ) in $25 \mathrm{ml} \mathrm{EtOH}$ were refluxed for $5 \mathrm{~min}$ under Ar. Then, $\mathrm{VOSO}_{4}(325 \mathrm{mg}, 1.5 \mathrm{mmol}$ ) was added and the mixture was refluxed for $50 \mathrm{~min}$. Next, the mixture was filtered, and bpy (236 $\mathrm{mg}, 1.5 \mathrm{mmol})$ was added to the filtrate. The complex was filtered off, washed three times with $\mathrm{EtOH}$, and dried in air. Yield $0.417 \mathrm{~g}, 51 \%$ $\mathrm{MW}=538.43$. Anal. Calc. for $\mathrm{C}_{25} \mathrm{H}_{21} \mathrm{~N}_{5} \mathrm{O}_{6} \mathrm{~V}: \mathrm{C}, 55.77 ; \mathrm{H}$, 3.93; N, 13.01\%. Found: C, 55.92; H, 3.62; N, 13.28\%. The complex is paramagnetic $\mu_{\mathrm{eff}}=1.51 \mu_{\mathrm{B}}$. FT-IR $\left(\mathrm{KBr}, \mathrm{cm}^{-1}\right)$ :
3429 (w, br), 3051 (w), 1600 (s), 1552 (w), 1511 (m), 1491 (m), 1466 (m), 1438 (m), 1357 (w), 1316 (vs), 1245 (w), 1201 (w), 1175 (w), 1101 (m), 1037 (w), 1023 (w), 957 (s), 906 (w), 828 (w), 801 (w), 767 (m), 734 (w), 717 (w), 701 (m), 653 (w), 563 (w), $524(\mathrm{w}), 454$ (w). UV-Vis (DMSO): 284, 327, 393 nm, (MeCN): 240, 304, 324, 389 nm, (EtOH): 286, $318,383 \mathrm{~nm}$.

\section{Preparation of $\left[\mathrm{VO}\left(\mathrm{L}_{1}\right)(\right.$ phen $\left.)\right] \cdot 1.5 \mathrm{H}_{2} \mathrm{O}(3)$}

5-Nitrosalicylaldehyde (500 $\mathrm{mg}, 3 \mathrm{mmol})$ and benzhydrazide (408 mg, $3 \mathrm{mmol}$ ) in $50 \mathrm{ml} \mathrm{EtOH}$ were refluxed for several minutes under Ar. Then, $\mathrm{VOSO}_{4}(666 \mathrm{mg}, 3 \mathrm{mmol}$ ) was added, and the mixture was refluxed for $40 \mathrm{~min}$. Next, the mixture was filtered and, to the $25 \mathrm{ml}$ of filtrate, phen (272.3 mg, $1.5 \mathrm{mmol}$ ) was added. The complex was filtered off, washed two times with $\mathrm{EtOH}$, and dried in air. Yield $0.651 \mathrm{~g}, 40 \% \mathrm{MW}=557.40$. Anal. Calc. for $\mathrm{C}_{26} \mathrm{H}_{21} \mathrm{~N}_{5} \mathrm{O}_{6.5} \mathrm{~V}$ : C, 56.02; H, 3.62; N, 12.56\%. Found: C, 56.32; H, 3.30; N, $12.30 \%$. The complex is paramagnetic $\mu_{\text {eff }}=1.61 \mu_{\mathrm{B}}$. FT-IR $\left(\mathrm{KBr}, \mathrm{cm}^{-1}\right.$ ): 3452 (w, br), 3051 (w), 1614 (s), 1598 (s), 1549 (w), 1510 (m), 1491 (w), 1465 (w), 1425 (w), 1360 (w), 1336 (w), 1315 (vs), 1243 (w), 1198 (w), $1141(\mathrm{w})$, 1102 (m), 1039 (w), 960 (m), 909 (w), 848 (w), 795 (w), 754 (w), $725(\mathrm{~m}), 703(\mathrm{~m}), 658(\mathrm{w}), 569(\mathrm{w}), 454(\mathrm{w}), 429(\mathrm{w})$. UV-Vis (DMSO): 326, $392 \mathrm{~nm},(\mathrm{MeCN}): 225,271,327$, $386 \mathrm{~nm},(\mathrm{EtOH}):$ 225, 269, 322, $394 \mathrm{~nm}$. 


\section{Preparation of $\left[\mathrm{VO}\left(\mathrm{L}_{2}\right)(\mathrm{EtO})\right](4)$}

5-Nitrosalicylaldehyde (250.7 $\mathrm{mg}, 1.5 \mathrm{mmol})$ and 2-hydroxybenzhydrazide (228.2 $\mathrm{mg}, 1.5 \mathrm{mmol}$ ) in $40 \mathrm{ml}$ $\mathrm{EtOH}$ were refluxed $15 \mathrm{~min}$ under $\mathrm{Ar}$. Then, $\mathrm{VOSO}_{4}$ (333 mg, $1.5 \mathrm{mmol}$ ) was added with additional $10 \mathrm{ml} \mathrm{EtOH,}$ and mixture was refluxed for $105 \mathrm{~min}$. Next, the mixture was filtered, washed with a small amount of $\mathrm{EtOH}$, and dried in air. Yield $0.356 \mathrm{~g}, 58 \% \mathrm{MW}=411.24$. Anal. Calc. for $\mathrm{C}_{16} \mathrm{H}_{14} \mathrm{~N}_{3} \mathrm{O}_{7} \mathrm{~V}: \mathrm{C}, 46.73 ; \mathrm{H}, 3.43 ; \mathrm{N}, 10.22 \%$. Found: $\mathrm{C}$, $46.58 ; \mathrm{H}, 3.66 ; \mathrm{N}, 10.08 \%$. The complex is diamagnetic. FT-IR (KBr, cm $\left.{ }^{-1}\right): 3482$ (w, br), 2981 (w), 1626 (vs), 1609 (vs), 1580 (w), 1560 (m), 1520 (s), 1484 (m), 1468 (m), 1381 (m), 1341 (vs), 1308 (w), 1284 (m), 1248 (m), 1218 (m), 1160 (m), 1128 (w), 1089 (m), 1065 (w), 1035 (m), $997(\mathrm{~s}), 966(\mathrm{w}), 908(\mathrm{~m}), 873(\mathrm{w}), 843(\mathrm{~m}), 817(\mathrm{~m}), 755$ (s), $720(\mathrm{~m}), 703(\mathrm{~m}), 674(\mathrm{w}), 656(\mathrm{w}), 614(\mathrm{~m}), 578(\mathrm{w})$, 519 (m), 481 (w), 468 (w), 447 (w). UV-Vis (DMSO): 326, $387 \mathrm{~nm},(\mathrm{MeCN})$ : 267, 310, 324, $389 \mathrm{~nm}$, (EtOH): $321 \mathrm{~nm}$.

\section{Preparation of $\left[\mathrm{VO}\left(\mathrm{L}_{2}\right)(\mathrm{bpy})\right] \cdot \mathrm{H}_{2} \mathrm{O}(5)$}

5-Nitrosalicylaldehyde $(250.7 \mathrm{mg}, 1.5 \mathrm{mmol})$ and 2-hydroxybenzhydrazide (228.2 $\mathrm{mg}, 1.5 \mathrm{mmol})$ in $33 \mathrm{ml}$ EtOH were refluxed for 6 min under Ar. Then, $\mathrm{VOSO}_{4}$ (333 mg, $1.5 \mathrm{mmol}$ ) was added, and the mixture was refluxed for $10 \mathrm{~min}$. Then, bpy ( $234 \mathrm{mg}, 1.5 \mathrm{mmol}$ ) in $2 \mathrm{ml} \mathrm{EtOH}$ was added. The mixture was refluxed for an additional $5 \mathrm{~min}$. The mixture was cooled, and the product was filtered off, washed with $\mathrm{EtOH}$, and dried in air. Yield $0.656 \mathrm{~g}, 81 \%$ $\mathrm{MW}=540.38$. Anal. Calc. for $\mathrm{C}_{24} \mathrm{H}_{19} \mathrm{~N}_{5} \mathrm{O}_{7} \mathrm{~V}: \mathrm{C}, 53.34 ; \mathrm{H}$, $3.54 ; \mathrm{N}, 12.96 \%$. Found: C, 53.57; H, 3.34; N, 13.00\%. The complex is paramagnetic $\mu_{\text {eff }}=1.46 \mu_{\mathrm{B}}$. FT-IR $\left(\mathrm{KBr}, \mathrm{cm}^{-1}\right)$ : 3476 (w, br), 3057 (w), 1604 (vs), 1551 (w), 1514 (m), 1491 (s), 1469 (m), 1443 (m), 1366 (m), 1315 (vs), 1248 (w), $1160(\mathrm{w}), 1130(\mathrm{w}), 1101(\mathrm{~m}), 1060(\mathrm{w}), 1022(\mathrm{w}), 955(\mathrm{~s})$, $914(\mathrm{w}), 830(\mathrm{w}), 802(\mathrm{w}), 753(\mathrm{~s}), 731(\mathrm{~m}), 720(\mathrm{~m}), 694$ (w), $653(\mathrm{~m}), 631(\mathrm{w}), 582(\mathrm{w}), 519(\mathrm{w}), 472(\mathrm{~m}), 446(\mathrm{w})$. UV-Vis (DMSO): 286, 329, 390, $465 \mathrm{~nm},(\mathrm{MeCN}): 214$, 306, $387 \mathrm{~nm}$, (EtOH): 284, $375 \mathrm{~nm}$.

\section{Preparation of $\left[\mathrm{VO}\left(\mathrm{L}_{3}\right)(\right.$ phen $\left.)\right] \cdot 2.5 \mathrm{H}_{2} \mathrm{O}(6)$}

5-Nitrosalicylaldehyde (250.7 $\mathrm{mg}, 1.5 \mathrm{mmol})$ and 4-hydroxybenzhydrazide ( $225.5 \mathrm{mg}, 1.5 \mathrm{mmol}$ ) in $40 \mathrm{ml}$ EtOH were refluxed 20 min under Ar. Then, $\left[\mathrm{VO}(\mathrm{acac})_{2}\right]$ (395 $\mathrm{mg}, 1.5 \mathrm{mmol}$ ) in $10 \mathrm{ml} \mathrm{EtOH}$ was added, and the mixture was refluxed for $30 \mathrm{~min}$. The mixture was filtered off, and to the filtrate, phen $(273.5 \mathrm{mg}, 1.5 \mathrm{mmol})$ in $10 \mathrm{ml} \mathrm{EtOH}$ was added. The mixture was heated for an additional $8 \mathrm{~min}$, and formation of orange precipitate was observed. This was filtered off, washed with a small amount of cold $\mathrm{EtOH}$, and dried in air. Yield $0.476 \mathrm{~g}, 54 \% \mathrm{MW}=591.42$. Anal. Calc. for $\mathrm{C}_{26} \mathrm{H}_{22} \mathrm{~N}_{5} \mathrm{O}_{8.5} \mathrm{~V}: \mathrm{C}, 52.80 ; \mathrm{H}, 3.75 ; \mathrm{N}, 11.84 \%$. Found: $\mathrm{C}, 52.80 ; \mathrm{H}, 3.34 ; \mathrm{N}, 11.67 \%$. The complex is paramagnetic $\mu_{\text {eff }}=1.28 \mu_{\mathrm{B}}$. FT-IR $\left(\mathrm{KBr}, \mathrm{cm}^{-1}\right): 3423(\mathrm{w}, \mathrm{br}), 1606$ (vs), 1554 (w), 1496 (vs), 1467 (w), 1425 (m), 1379 (m), 1361 (m), 1334 (s), 1313 (vs), 1244 (w), 1197 (w), 1166 (m), 1143 (w), $1103(\mathrm{~m}), 1033(\mathrm{w}), 956(\mathrm{~s}), 913(\mathrm{w}), 846(\mathrm{~s}), 757(\mathrm{~m})$, $724(\mathrm{~s}), 645(\mathrm{~m}), 597(\mathrm{w}), 566(\mathrm{w}), 521(\mathrm{w}), 475(\mathrm{w}), 445$ (w). UV-Vis (DMSO): 326, $395 \mathrm{~nm},(\mathrm{MeCN}): 223,270$, 328, $392 \mathrm{~nm}$, (EtOH): 226, 266, 326, $390 \mathrm{~nm}$.

\section{Preparation of $\left[\mathrm{VO}\left(\mathrm{L}_{4}\right)(\mathrm{EtO})\right] \cdot \mathrm{H}_{2} \mathrm{O}(7)$}

5-Nitrosalicylaldehyde $(251.0 \mathrm{mg}, 1.5 \mathrm{mmol})$ and phenylacetic acid hydrazide $(226.1 \mathrm{mg}, 1.5 \mathrm{mmol})$ in $67 \mathrm{ml}$ EtOH were refluxed 20 min under Ar. Then, [VO(acac $)_{2}$ ] $(399.0 \mathrm{mg}, 1.5 \mathrm{mmol}$ ) was added, and the mixture was refluxed for $42 \mathrm{~min}$. This was then evaporated to ca. $20 \mathrm{ml}$ and left aside for crystallization. The next day, brown crystalline product was filtered off, washed with $\mathrm{EtOH}$, and dried in air. Yield $0.340 \mathrm{~g}, 53 \% \mathrm{MW}=427.28$. Anal. Calc. for $\mathrm{C}_{17} \mathrm{H}_{18} \mathrm{~N}_{3} \mathrm{O}_{5} \mathrm{~V}: \mathrm{C}, 47.79 ; \mathrm{H}, 4.25 ; \mathrm{N}, 9.83 \%$. Found: $\mathrm{C}$, 47.46; H, 4.14; N, 9.69\%. The complex is diamagnetic. FT-IR (KBr, cm $\left.{ }^{-1}\right): 3547$ (m), 2974 (w), 1612 (s), 1598 (s), 1557 (w), 1526 (s), 1497 (s), 1461 (w), 1334 (vs), 1303 (s), $1201(\mathrm{w}), 1155(\mathrm{w}), 1128(\mathrm{w}), 1088(\mathrm{~m}), 1031(\mathrm{~m}), 983(\mathrm{~s})$, $941(\mathrm{w}), 909(\mathrm{~m}), 841(\mathrm{~m}), 808(\mathrm{w}), 781(\mathrm{w}), 759(\mathrm{w}), 723$ (m), $692(\mathrm{w}), 660(\mathrm{w}), 635(\mathrm{~m}), 582(\mathrm{~m}), 549(\mathrm{w}), 513(\mathrm{w})$, 460 (m), 429 (w). UV-Vis (DMSO): $319 \mathrm{~nm},(\mathrm{MeCN}): 289$, $334 \mathrm{~nm}$, (EtOH): 308, $364 \mathrm{~nm}$.

\section{Preparation of $\left[\mathrm{VO}\left(\mathrm{L}_{4}\right)(\right.$ phen $\left.)\right](8)$}

The synthesis was performed for $\mathbf{6}$. The hydrazide used was phenylacetic acid hydrazide $(227.1 \mathrm{mg}, 1.5 \mathrm{mmol})$. Yield $0.649 \mathrm{~g}, 79 \% \mathrm{MW}=544.41$. Anal. Calc. for $\mathrm{C}_{27} \mathrm{H}_{19} \mathrm{~N}_{5} \mathrm{O}_{5} \mathrm{~V}$ : C, 59.57; H, 3.52; N, 12.86\%. Found: C, 59.20; H, 3.51; N, $12.62 \%$. The complex is paramagnetic $\mu_{\text {eff }}=1.47 \mu_{\mathrm{B}}$. FT-IR $\left(\mathrm{KBr}, \mathrm{cm}^{-1}\right.$ ): 3446 (w, br), 3057 (w), 1616 (s), 1601 (s), 1551 (m), 1520 (vs), 1489 (s), 1467 (w), 1425 (s), 1365 (m), 1313 (vs), 1250 (w), 1197 (w), 1143 (w), 1102 (m), $1028(\mathrm{w}), 957$ (s), 941 (m), 908 (w), $840(\mathrm{~s}), 800$ (w), 759 (w), $722(\mathrm{~s}), 689(\mathrm{w}), 661(\mathrm{w}), 647(\mathrm{w}), 587(\mathrm{w}), 558(\mathrm{w})$, $539(\mathrm{w}), 517(\mathrm{w}), 473(\mathrm{~m}), 428(\mathrm{w}) . \mathrm{UV}-\mathrm{Vis}(\mathrm{DMSO}): 313$, $380 \mathrm{~nm},(\mathrm{MeCN}): 270,316,375 \mathrm{~nm},(\mathrm{EtOH}): 362 \mathrm{~nm}$.

\section{Preparation of $\left[\mathrm{VO}\left(\mathrm{L}_{5}\right)\left(\mathrm{H}_{2} \mathrm{O}\right)\right](9)$}

5-Nitrosalicylaldehyde (251 mg, $1.5 \mathrm{mmol})$ and nicotinic acid hydrazide (206 mg, $1.5 \mathrm{mmol}$ ) in $70 \mathrm{ml} \mathrm{EtOH}$ were refluxed for $10 \mathrm{~min}$ under $\mathrm{Ar}$. Then, [VO(acac $\left.)_{2}\right](398 \mathrm{mg}$, $1.5 \mathrm{mmol}$ ) was added, and the mixture was refluxed for $20 \mathrm{~min}$. The obtaining product was filtered off, washed with small amount of EtOH, and dried in air. Yield $0.479 \mathrm{~g}, 75 \%$ 
$\mathrm{MW}=369.18$. Anal. Calc. for $\mathrm{C}_{13} \mathrm{H}_{10} \mathrm{~N}_{4} \mathrm{O}_{6} \mathrm{~V}: \mathrm{C}, 42.29 ; \mathrm{H}$, $2.73 ; \mathrm{N}, 15.18 \%$. Found: $\mathrm{C}, 42.31 ; \mathrm{H}, 2.72 ; \mathrm{N}, 14.85 \%$. The complex is paramagnetic $\mu_{\mathrm{eff}}=1.39 \mu_{\mathrm{B}}$. FT-IR $\left(\mathrm{KBr}, \mathrm{cm}^{-1}\right)$ : 3417 (w, br), 3070 (w), 1605 (s), 1551 (m), 1520 (m), 1500 (w), 1465 (m), 1365 (m), 1337 (vs), 1312 (m), 1242 (w), 1197 (m), 1154 (w), 1127 (w), 1099 (m), 1056 (w), 1027 (w), $957(\mathrm{w}), 919(\mathrm{w}), 880(\mathrm{vs}), 834(\mathrm{w}), 800(\mathrm{w}), 755(\mathrm{w})$, $721(\mathrm{~m}), 694(\mathrm{~m}), 656(\mathrm{~m}), 579(\mathrm{~m}), 517(\mathrm{w}), 454(\mathrm{~m})$. UV-Vis (DMSO): 324, $391 \mathrm{~nm},(\mathrm{MeCN}): 378,317 \mathrm{~nm}$, (EtOH): $319 \mathrm{~nm}$.

\section{Preparation of $\left[\mathrm{VO}\left(\mathrm{L}_{6}\right)(\mathrm{EtO})(\mathrm{EtOH})\right](10)$}

5-Nitrosalicylaldehyde (334 mg, $2 \mathrm{mmol}$ ) and 3-hydroxy2-naphthoic acid hydrazide (404 mg, $2 \mathrm{mmol}$ ) in $110 \mathrm{ml}$ $\mathrm{EtOH}$ were heated on a magnetic stirrer for 15 min under Ar. The temperature was raised to $100{ }^{\circ} \mathrm{C}$. Then, $\mathrm{VOSO}_{4}$ (444 mg, $2 \mathrm{mmol}$ ) and $20 \mathrm{ml}$ ethanol was added, and heterogenic mixture was refluxed for ca. $40 \mathrm{~min}$. After that, the volume of the reaction mixture was concentrated to final volume of ca. $40 \mathrm{ml}$ and cooled to room temperature. The precipitate was filtered off, washed with ethanol, and dried in air. Yield $0.652 \mathrm{~g}, 65 \% \mathrm{MW}=507.37$. Anal. Calc. for $\mathrm{C}_{22} \mathrm{H}_{22} \mathrm{~N}_{3} \mathrm{O}_{8} \mathrm{~V}$ : C, 52.08; H, 4.37; N, 8.28\%. Found: C, $51.58 ; \mathrm{H}, 4.21 ; \mathrm{N}, 8.17 \%$. The complex is diamagnetic. FT-IR (KBr, cm ${ }^{-1}$ ): 3435 (w, br), 3075 (w), 1633 (s), 1612 (s), 1555 (s), 1524 (vs), 1512 (s), 1458 (m), 1378 (m), 1341 (vs), 1302 (w), 1263 (w), 1239 (w), 1198 (w), 1175 (w), $1149(\mathrm{~m}), 1128(\mathrm{w}), 1089(\mathrm{~m}), 956(\mathrm{w}), 905$ (s), $884(\mathrm{w})$, $845(\mathrm{w}), 804(\mathrm{w}), 757(\mathrm{~m}), 710(\mathrm{w}), 644(\mathrm{~m}), 591(\mathrm{w}), 570$ (w), $519(\mathrm{w}), 481(\mathrm{~m}), 423(\mathrm{w})$. UV-Vis (DMSO): 326, $391 \mathrm{~nm},(\mathrm{MeCN}): 358,415 \mathrm{~nm},(\mathrm{EtOH}): 324,454 \mathrm{~nm}$.

\section{Preparation of $\left[\mathrm{VO}\left(\mathrm{HL}_{7}\right)_{2}\right] \cdot$ EtOH (11)}

5-Methoxysalicylaldehyde (187 $\mu \mathrm{l}, 1.5 \mathrm{mmol})$ and benzhydrazide $(207.3 \mathrm{mg}, 1.5 \mathrm{mmol}$ ) in $25 \mathrm{ml} \mathrm{EtOH}$ were refluxed for $12 \mathrm{~min}$ under Ar. Then, [VO(acac) $\left.{ }_{2}\right](398.6 \mathrm{mg}$, $1.5 \mathrm{mmol}$ ) was added, and the mixture was refluxed for the next $45 \mathrm{~min}$. After that, the solution was evaporated to ca. $10 \mathrm{ml}$ and left cooling in the refrigerator. The product was filtered off, washed with ethanol, and dried in air. Yield $0.170 \mathrm{~g}, 35 \% \mathrm{MW}=650.55$. Anal. Calc. for $\mathrm{C}_{32} \mathrm{H}_{31} \mathrm{~N}_{4} \mathrm{O}_{8} \mathrm{~V}$ : C, 59.17; H, 4.80; N, 8.63\%. Found: C, 59.73; H, 4.62; N, $8.50 \%$. The complex is paramagnetic $\mu_{\text {eff }}=1.27 \mu_{\mathrm{B}}$. FT-IR $\left(\mathrm{KBr}, \mathrm{cm}^{-1}\right)$ : 3464 (w, br), 3053 (w), 2915 (w), 2833 (w), 1603 (w), 1575 (m), 1539 (vs), 1503 (m), 1454 (m), 1440 (m), 1410 (w), 1356 (s), 1327 (m), 1275 (s), 1225 (vs), 1196 (w), 1169 (m), 1137 (w), 1119 (w), 1031 (s), 945 (w), 830 (m), $810(\mathrm{~m}), 785(\mathrm{w}), 738(\mathrm{w}), 697(\mathrm{~s}), 613(\mathrm{w}), 586(\mathrm{w})$, 553 (w), 516 (w), 484 (w). UV-Vis (DMSO): $300,396 \mathrm{~nm}$, (MeCN): 297, 386, 519 nm, (EtOH): 296, 399, $521 \mathrm{~nm}$.

\section{Preparation of $\left[\left\{\mathrm{VO}\left(\mathrm{HL}_{7}\right)\right\}_{2}\left(\mathrm{SO}_{4}\right)\right] \cdot \mathrm{H}_{2} \mathrm{O}(12)$}

5-Methoxysalicylaldehyde (187 $\mu \mathrm{l}, 1.5 \mathrm{mmol})$ and benzhydrazide ( $207 \mathrm{mg}, 1.5 \mathrm{mmol}$ ) in $25 \mathrm{ml} \mathrm{EtOH}$ were refluxed for $10 \mathrm{~min}$ under Ar. Then, $\mathrm{VOSO}_{4}(331.4 \mathrm{mg}, 1.5 \mathrm{mmol})$ was added, and the mixture was refluxed for the next $40 \mathrm{~min}$. After that, the solution was evaporated to ca. $10 \mathrm{ml}$ and left cooling in the refrigerator. The product was filtered off, washed with ethanol, and dried in air. Yield $0.402 \mathrm{~g}, 68 \%$ $\mathrm{MW}=788.51$. Anal. Calcd. for $\mathrm{C}_{30} \mathrm{H}_{30} \mathrm{~N}_{4} \mathrm{O}_{13} \mathrm{SV}_{2}$ : C, 45.81; H, 3.80; N, 7.12; S, 4.08\%. Found: C, 45.77; H, 4.65; N, 7.10; $\mathrm{S} \sim 4 \%$. The complex is paramagnetic, $\mu=1.27 \mu_{\mathrm{B}}$. FT-IR (KBr, cm $\left.{ }^{-1}\right)$ : 3423 (w, br), $2945(w), 2845(w), 1617$ (w), $1605(\mathrm{w}), 1581(\mathrm{w}), 1537$ (s), $1490(\mathrm{~s}), 1448$ (w), 1417 (w), 1388 (s), 1346 (s), $1284(\mathrm{~m}), 1268$ (w), 1243 (s), 1171 (s), 1121 (vs), 1091 (s), 1030 (s), 1002 (m), 968 (s), 897 (m), $828(\mathrm{~m}), 793(\mathrm{w}), 700(\mathrm{~s}), 671(\mathrm{w}), 629(\mathrm{w}), 609(\mathrm{w})$, $584(\mathrm{w}), 560(\mathrm{w}), 507$ (w), 471 (w). UV-Vis (DMSO): 331, $424 \mathrm{~nm},(\mathrm{MeCN}): 326,419 \mathrm{~nm},(\mathrm{EtOH}): 327,416 \mathrm{~nm}$.

\section{Preparation of $\left[\mathrm{VO}\left(\mathrm{L}_{8}\right)(\right.$ phen $\left.)\right] \cdot 4 \mathrm{H}_{2} \mathrm{O}(13)$}

5-Methoxysalicylaldehyde $(187 \mu \mathrm{l}, 1.5 \mathrm{mmol})$ and 2-hydroxybenzhydrazide ( $227.4 \mathrm{mg}, 1.5 \mathrm{mmol})$ in $25 \mathrm{ml}$ EtOH were refluxed for 10 min under Ar. Then, $\mathrm{VOSO}_{4}$ (334 mg, $1.5 \mathrm{mmol}$ ) was added, and the mixture was refluxed for the next $47 \mathrm{~min}$. Phen $(272.9 \mathrm{mg}, 1.5 \mathrm{mmol})$ was added to the transparent solution, and heating was continued for $10 \mathrm{~min}$. This slowly started to crystallize, especially on cooling. The brown crystalline product was filtered off, washed with EtOH, and dried in air. Yield $0.369 \mathrm{~g}, 38 \%$ $\mathrm{MW}=$ 703.48. Anal. Calc. for $\mathrm{C}_{27} \mathrm{H}_{28} \mathrm{~N}_{4} \mathrm{O}_{9} \mathrm{~V}: \mathrm{C}, 53.74 ; \mathrm{H}$, 4.68; N, 9.28\%. Found: C, 53.56; H, 3.86; N, 9.31\%. The complex is paramagnetic $\mu_{\text {eff }}=1.41 \mu_{\mathrm{B}}$. FT-IR $\left(\mathrm{KBr}, \mathrm{cm}^{-1}\right)$ : 3429 (m, br), 3064 (w), 1623 (m), 1596 (s), 1537 (s), 1518 (s), 1491 (s), $1466(\mathrm{w}), 1424$ (s), $1374(\mathrm{~m}), 1340$ (w), 1286 (w), $1257(\mathrm{~m}), 1223(\mathrm{~m}), 1166(\mathrm{~m}), 1145(\mathrm{w}), 1107$ (w), $1032(\mathrm{~s}), 957(\mathrm{~s}), 867(\mathrm{w}), 846(\mathrm{~m}), 816(\mathrm{w}), 759(\mathrm{~m}), 722$ (s), 699 (w), $657(\mathrm{w}), 643(\mathrm{w}), 582(\mathrm{w}), 473(\mathrm{w}), 449(\mathrm{w})$, 427 (w). UV-Vis (DMSO): 331, $429 \mathrm{~nm},(\mathrm{MeCN}): 297,386$, $519 \mathrm{~nm},(\mathrm{EtOH}): 267,339,439 \mathrm{~nm}$.

\section{Preparation of $\left[\mathrm{VO}\left(\mathrm{L}_{9}\right)(\right.$ phen $\left.)\right] \cdot 1.5 \mathrm{H}_{2} \mathrm{O}(14)$}

The synthesis was performed as for $\mathbf{6}$. The aldehyde used was 5 -methoxysalicylaldehyde $(187 \mu 1,1.5 \mathrm{mmol})$, and the hydrazide used was 4-hydroxybenzhydrazide $(226.7 \mathrm{mg}$, $1.5 \mathrm{mmol}$ ). Yield $0.359 \mathrm{~g}, 43 \% \mathrm{MW}=558.44$. Anal. Calc. for $\mathrm{C}_{27} \mathrm{H}_{23} \mathrm{~N}_{4} \mathrm{O}_{6.5} \mathrm{~V}$ : C, 58.07; H, 4.15; N, 10.03\%. Found: $\mathrm{C}, 58.25 ; \mathrm{H}, 4.06 ; \mathrm{N}, 9.77 \%$. The complex is paramagnetic $\mu_{\text {eff }}=1.60 \mu_{\mathrm{B}}$. FT-IR $\left(\mathrm{KBr}, \mathrm{cm}^{-1}\right): 3418$ (w, br), 3163 (w, br), 
1598 (vs), 1545 (s), 1501 (vs), 1477 (m), 1423 (m), 1374 (w), $1360(\mathrm{~m}), 1339(\mathrm{w}), 1279(\mathrm{~s}), 1226(\mathrm{~s}), 1169(\mathrm{~s}), 1104$ (w), $1031(\mathrm{~m}), 954(\mathrm{~s}), 906(\mathrm{w}), 845(\mathrm{~s}), 810(\mathrm{~m}), 787(\mathrm{w})$, 757 (w), 724 (m), 697 (w), $642(\mathrm{w}), 597(\mathrm{w}), 562(\mathrm{w}), 474$ (w), 441 (w). UV-Vis (DMSO): 334, $426 \mathrm{~nm},(\mathrm{MeCN}): 269$, 334, $428 \mathrm{~nm}$, (EtOH): 228, 264, 330, $421 \mathrm{~nm}$.

\section{Preparation of $\left[\mathrm{VO}\left(\mathrm{L}_{10}\right)(\right.$ phen $\left.)\right](15)$}

The synthesis was performed as for $\mathbf{1 4}$. The hydrazide used was 4-chlorobenzhydrazide $(252.1 \mathrm{mg}, 1.5 \mathrm{mmol})$. Yield $0.565 \mathrm{~g}, 69 \% \mathrm{MW}=549.86$. Anal. Calc. for $\mathrm{C}_{27} \mathrm{H}_{19} \mathrm{ClN}_{4} \mathrm{O}_{4} \mathrm{~V}: \mathrm{C}, 58.98 ; \mathrm{H}, 3.48 ; \mathrm{N}, 10.19 \%$. Found: $\mathrm{C}$, $58.91 ; \mathrm{H}, 3.47 ; \mathrm{N}, 10.15 \%$. The complex is paramagnetic $\mu_{\text {eff }}=1.53 \mu_{\mathrm{B}}$. FT-IR $\left(\mathrm{KBr}, \mathrm{cm}^{-1}\right): 3452(\mathrm{w}, \mathrm{br}), 3051(\mathrm{w})$, 2821 (w), 1623 (w), 1602 (s), 1543 (s), 1506 (vs), 1486 (s), $1466(\mathrm{~m}), 1423(\mathrm{~s}), 1375(\mathrm{~m}), 1348(\mathrm{~m}), 1293(\mathrm{~m})$, 1260 (s), 1222 (w), 1179 (w), 1159 (m), 1089 (w), 1053 (w), $1030(\mathrm{~m}), 1012(\mathrm{w}), 955(\mathrm{vs}), 906(\mathrm{w}), 867(\mathrm{w}), 851$ (m), $811(\mathrm{~m}), 774(\mathrm{w}), 741$ (w), $729(\mathrm{~m}), 680(\mathrm{w}), 644$ (w), $583(\mathrm{w}), 467$ (w), $436(\mathrm{w})$. UV-Vis (DMSO): 331, $428 \mathrm{~nm},(\mathrm{MeCN}): 267,337,447 \mathrm{~nm},(\mathrm{EtOH}): 228,264$, $326,430 \mathrm{~nm}$.

\section{Preparation of $\left[\mathrm{VO}\left(\mathrm{L}_{11}\right)(\mathrm{EtO})(\mathrm{EtOH})\right](16)$}

5-Methoxysalicylaldehyde (187 $\mu \mathrm{l}, 1.5 \mathrm{mmol})$ and phenylacetic acid hydrazide $(225.2 \mathrm{mg}, 1.5 \mathrm{mmol})$ in $50 \mathrm{ml} \mathrm{EtOH}$ were refluxed for $11 \mathrm{~min}$ under Ar. Then, [VO(acac $)_{2}$ ] (399 mg, $1.5 \mathrm{mmol}$ ) was added, and the mixture was refluxed for the next $58 \mathrm{~min}$. This was evaporated in part, but precipitation was observed the next day in refrigerator. This was filtered off, washed with $\mathrm{EtOH}$, and dried in air. Yield 0.410 g, $62 \% \mathrm{MW}=440.36$. Anal. Calc. for $\mathrm{C}_{20} \mathrm{H}_{25} \mathrm{~N}_{2} \mathrm{O}_{6} \mathrm{~V}$ : C, 54.67; H, 5.68; N, 6.38\%. Found: C, 54.55; H, 5.72; N, $6.36 \%$. The complex is diamagnetic. FT-IR $\left(\mathrm{KBr}, \mathrm{cm}^{-1}\right)$ : 3452 (w, br), 2990 (w), 1625 (w) 1600 (s), 1546 (vs), 1514 (vs), 1468 (m), 1421 (m), 1377 (w), 1358 (m), $1326(\mathrm{w})$, 1288 (m), 1265 (vs), 1238 (w), 1181 (w), 1160 (m), 1107 (w), $1048(\mathrm{w}), 1026(\mathrm{~m}), 991$ (vs), $901(\mathrm{w}), 844(\mathrm{~m}), 817$ (m), 766(w), $726(\mathrm{vs}), 690(\mathrm{w}), 637(\mathrm{w}), 587(\mathrm{w}), 553(\mathrm{w})$, $524(\mathrm{w}), 467$ (w), $425(\mathrm{w})$. UV-Vis (DMSO): $323,413 \mathrm{~nm}$, $(\mathrm{MeCN}): 408 \mathrm{~nm},(\mathrm{EtOH}): 315,412 \mathrm{~nm}$.

\section{Preparation of $\left[\mathrm{VO}\left(\mathrm{L}_{11}\right)(\right.$ phen $\left.)\right](17)$}

The synthesis was performed as for $\mathbf{1 4}$. The hydrazide used was phenylacetic acid hydrazide $(225.8 \mathrm{mg}, 1.5 \mathrm{mmol})$. Yield $0.619 \mathrm{~g}, 78 \% \mathrm{MW}=529.44$. Anal. Calc. for $\mathrm{C}_{28} \mathrm{H}_{22} \mathrm{~N}_{4} \mathrm{O}_{4} \mathrm{~V}: \mathrm{C}, 63.52 ; \mathrm{H}, 4.19 ; \mathrm{N}, 10.58 \%$. Found: $\mathrm{C}$, $63.26 ; \mathrm{H}, 4.11 ; \mathrm{N}, 10.43 \%$. The complex is paramagnetic $\mu_{\mathrm{eff}}=1.59 \mu_{\mathrm{B}}$. FT-IR $\left(\mathrm{KBr}, \mathrm{cm}^{-1}\right): 3135(\mathrm{w}, \mathrm{br}), 2966(\mathrm{w})$, 1602 (s), 1550 (vs), 1531 (vs), 1476 (s), 1455 (m), 1419 (w), 1383 (w), 1330 (w), 1270 (vs), 1230 (s), 1194 (w), 1164 (m), 1088 (w), 1048 (vs), 1025 (m), 974 (vs), 920 (m), 878 (w), 836 (s), 774 (w), 736 (s), $686(\mathrm{w}), 663$ (m), 580 (w), 564 (w), $524(\mathrm{w}), 473(\mathrm{w}), 444(\mathrm{w}), 425$ (w). UV-Vis (DMSO): 412, $396 \mathrm{~nm}$, (EtOH): $262 \mathrm{~nm}$.

\section{Preparation of $\left[\mathrm{VO}\left(\mathrm{L}_{12}\right)\left(\mathrm{H}_{2} \mathrm{O}\right)\right] \cdot 0.5 \mathrm{H}_{2} \mathrm{O}(18)$}

5-Methoxysalicylaldehyde ( $374 \mu \mathrm{l}, 3 \mathrm{mmol})$ and nicotinic acid hydrazide $(411 \mathrm{mg}, 3 \mathrm{mmol}$ ) in $45 \mathrm{ml} \mathrm{EtOH}$ were refluxed for $15 \mathrm{~min}$ under Ar. Then, $\mathrm{VOSO}_{4}(666 \mathrm{mg}$, $3 \mathrm{mmol}$ ) was added, and the mixture was refluxed for the next $40 \mathrm{~min}$. The compound was filtered off, washed with $\mathrm{EtOH}$, and dried in air. Yield $0.800 \mathrm{~g}, 73 \% \mathrm{MW}=363.20$. Anal. Calc. for $\mathrm{C}_{14} \mathrm{H}_{14} \mathrm{~N}_{3} \mathrm{O}_{5.5} \mathrm{~V}: \mathrm{C}, 46.26 ; \mathrm{H}, 3.85 ; \mathrm{N}$, $11.56 \%$. Found: $\mathrm{C}, 46.06 ; \mathrm{H}, 3.22 ; \mathrm{N}, 11.29 \%$. The complex is paramagnetic $\mu_{\mathrm{eff}}=1.42 \mu_{\mathrm{B}}$. FT-IR $\left(\mathrm{KBr}, \mathrm{cm}^{-1}\right): 3490(\mathrm{w}$, br), $3066(\mathrm{w}), 1625(\mathrm{~m}), 1597$ (s), 1551 (vs), 1481 (s), 1461 (m), $1420(\mathrm{w}), 1340(\mathrm{~s}), 1287(\mathrm{~s}), 1260(\mathrm{w}), 1219$ (s), 1195 (m), $1171(\mathrm{~s}), 1031(\mathrm{~s}), 949(\mathrm{~s}), 932(\mathrm{~m}), 872(\mathrm{~s}), 827(\mathrm{~s})$, $786(\mathrm{~m}), 707$ (s), $673(\mathrm{~m}), 614(\mathrm{~s}), 574(\mathrm{~s}), 477(\mathrm{~m})$. UV-Vis (DMSO): 333, $429 \mathrm{~nm},(\mathrm{MeCN}): 254 \mathrm{~nm},(\mathrm{EtOH}): 249 \mathrm{~nm}$.

\section{Preparation of $\left[\mathrm{VO}\left(\mathrm{L}_{13}\right)(\mathrm{EtO})\right] \cdot 0.5 \mathrm{H}_{2} \mathrm{O}(19)$}

5-Methoxysalicylaldehyde (187 $\mu \mathrm{l}, 1.5 \mathrm{mmol})$ and 3-hydroxy-2-naphthoic acid hydrazide (303 mg, $1.5 \mathrm{mmol}$ ) in $90 \mathrm{ml} \mathrm{EtOH}$ were heated on a magnetic stirrer for $10 \mathrm{~min}$ under Ar. The temperature was raised to $100{ }^{\circ} \mathrm{C}$. Then, $\mathrm{VOSO}_{4}(333 \mathrm{mg}, 1.5 \mathrm{mmol})$ and $10 \mathrm{ml}$ of $\mathrm{EtOH}$ was added, and the heterogenic mixture was refluxed for ca. $180 \mathrm{~min}$. After that, the volume of the reaction mixture was concentrated to a final volume of ca. $40 \mathrm{ml}$ and cooled to room temperature. The precipitate was filtered off, washed with ethanol, and dried in air. Yield $0.698 \mathrm{~g}, 78 \% \mathrm{MW}=446.33$. Anal. Calc. for $\mathrm{C}_{21} \mathrm{H}_{19} \mathrm{~N}_{2} \mathrm{O}_{6} \mathrm{~V}$ : C, 56.51; H, 4.29; N, 6.28\%. Found: $\mathrm{C}, 56.08 ; \mathrm{H}, 4.25 ; \mathrm{N}, 6.15 \%$. The complex is diamagnetic. FT-IR $\left(\mathrm{KBr}, \mathrm{cm}^{-1}\right)$ : 3453 (w, br), 2932 (w), 1639 (m), 1596 (m), 1572 (w), 1554 (s), 1524 (m), 1473 (m), $1386(\mathrm{w}), 1371(\mathrm{w}), 1341(\mathrm{w}), 1309(\mathrm{~m}), 1274(\mathrm{~s}), 1239(\mathrm{~m})$, $1212(\mathrm{w}), 1168(\mathrm{w}), 1145(\mathrm{w}), 1088(\mathrm{~m}), 1031(\mathrm{~s}), 991(\mathrm{~s})$, $954(\mathrm{w}), 904(\mathrm{w}), 822(\mathrm{~m}), 765(\mathrm{w}), 719(\mathrm{w}), 683(\mathrm{w}), 622$ (m), $597(\mathrm{~m}), 577(\mathrm{~m}), 527(\mathrm{w}), 478(\mathrm{w}) . \mathrm{UV}-\mathrm{Vis}$ (DMSO): 333, $438 \mathrm{~nm},(\mathrm{MeCN}): 325,427 \mathrm{~nm},(\mathrm{EtOH}): 321,428 \mathrm{~nm}$.

\section{Preparation of $\left[\mathrm{VO}\left(\mathrm{L}_{14}\right)(\right.$ phen $\left.)\right] \cdot 4.5 \mathrm{H}_{2} \mathrm{O}(20)$}

Potassium salt of 5-sulfosalicylaldehyde (366 mg, $1.5 \mathrm{mmol}$ ) and 4-hydroxybenzhydrazide (228 $\mathrm{mg}, 1.5 \mathrm{mmol})$ in $50 \mathrm{ml}$ ethanol were refluxed for $10 \mathrm{~min}$ under Ar. Then, [VO(acac $\left.)_{2}\right](397 \mathrm{mg}, 1.5 \mathrm{mmol})$ was added. The reflux 
was continued for $15 \mathrm{~min}$ under Ar. Next, phen $(273 \mathrm{mg}$, $1.5 \mathrm{mmol}$ ) in $10 \mathrm{ml}$ of EtOH was added, and the mixture was heated for an additional $8 \mathrm{~min}$. The next day, formed crystals were filtered off, washed with cold ethanol, and dried in air. Yield: $0.312 \mathrm{~g}, 30 \%$. $\mathrm{MW}=700.60$. Anal. Calcd. for $\mathrm{C}_{26} \mathrm{H}_{27} \mathrm{~N}_{4} \mathrm{O}_{11.5} \mathrm{KSV}$ : C, 44.57; H, 3.74; N, 5.58; S, 4.64\%. Found: C, 44.70; H, 3.54; N, 7.85; S, 4.31\%. The complex is paramagnetic, $\mu_{\mathrm{eff}}=1.26 \mu_{\mathrm{B}}$. FT-IR $\left(\mathrm{KBr}, \mathrm{cm}^{-1}\right): 3436$ (vs), 1606 (vs), 1501 (s), 1463 (w), 1423 (m), 1375 (m), 1356 (m), $1280(\mathrm{w}), 1194(\mathrm{w}), 1166(\mathrm{~s}), 1112(\mathrm{~m}), 1033$ (vs), 958 (m), $903(\mathrm{w}), 844(\mathrm{~m}), 723(\mathrm{~m}), 673(\mathrm{w}), 648(\mathrm{w}), 617(\mathrm{~m})$, 565 (w), 450 (w). UV-Vis (DMSO): 327, $407 \mathrm{~nm},(\mathrm{MeCN})$ : 268, 330, 414 nm, (EtOH): 226, 264, 325, $406 \mathrm{~nm}$.

\section{Preparation of $\left[\mathrm{VO}\left(\mathrm{L}_{15}\right)(\right.$ phen $\left.)\right] \cdot 4 \mathrm{H}_{2} \mathrm{O}(21)$}

Potassium salt of 5-sulfosalicylaldehyde ( $367 \mathrm{mg}, 1.5 \mathrm{mmol}$ ) and 4-methoxybenzhydrazide $(250 \mathrm{mg}, 1.5 \mathrm{mmol})$ in $45 \mathrm{ml}$ ethanol were refluxed for $20 \mathrm{~min}$ under Ar. Then, $\left[\mathrm{VO}(\mathrm{acac})_{2}\right](378 \mathrm{mg}, 1.5 \mathrm{mmol})$ was added. The reflux was continued for $20 \mathrm{~min}$ under Ar. Next, phen $(271 \mathrm{mg}$, $1.5 \mathrm{mmol}$ ) was added. The mixture with precipitate was evaporated to $50 \%$ and left for cooling. The mixture was filtered off, washed with ethanol, and dried in air. Yield: $0.599 \mathrm{~g}, 55 \%$. $\mathrm{MW}=705.63$. Anal. Calcd. for $\mathrm{C}_{27} \mathrm{H}_{27} \mathrm{~N}_{4} \mathrm{O}_{11} \mathrm{KSV}$ : C, 45.96; H, 3.86; N, 7.94; S, 4.54\%. Found: C, 45.65; H, 3.70; N, 7.83; S, 4.40\%. The complex is paramagnetic, $\mu_{\mathrm{eff}}=1.34 \mu_{\mathrm{B}}$. FT-IR $\left(\mathrm{KBr}, \mathrm{cm}^{-1}\right): 3436$ (vs), 1609 (vs), 1535 (w), 1499 (s), 1461 (m), 1421 (m), $1371(\mathrm{~m}), 1352(\mathrm{~s}), 1310(\mathrm{w}), 1252$ (s), 1175 (vs), $1116(\mathrm{~s})$, 1040 (vs), 963 (s), $905(\mathrm{w}), 846(\mathrm{~m}), 751(\mathrm{w}), 728(\mathrm{~m}), 675$ (m), $646(\mathrm{w}), 617(\mathrm{~m}), 587(\mathrm{w}), 469(\mathrm{w}), 437(\mathrm{w}) . \mathrm{UV}-\mathrm{Vis}$ (DMSO): 325, $406 \mathrm{~nm},(\mathrm{MeCN}): 224,268,327,420 \mathrm{~nm}$, (EtOH): 226, 263, 325, $406 \mathrm{~nm}$.

\section{Preparation of $\left[\mathrm{VO}\left(\mathrm{L}_{16}\right)(\right.$ phen $\left.)\right] \cdot$ phen $\cdot 2 \mathrm{H}_{2} \mathrm{O}(22)$}

Potassium salt of 5-sulfosalicylaldehyde ( $361 \mathrm{mg}, 1.5 \mathrm{mmol}$ ) and phenylacetic acid hydrazide $(225 \mathrm{mg}, 1.5 \mathrm{mmol})$ in $30 \mathrm{ml}$ ethanol were refluxed for $10 \mathrm{~min}$ under Ar. Then, [VO(acac) $)_{2}$ ( $(398 \mathrm{mg}, 1.5 \mathrm{mmol})$ was added. The reflux was continued for $45 \mathrm{~min}$ under Ar. Then, phen $(270 \mathrm{mg}$, $1.5 \mathrm{mmol}$ ) was added, and reflux was continued for $5 \mathrm{~min}$. The formed precipitate was filtered off, washed with ethanol, and dried in air. Yield: $0.493 \mathrm{~g}, 42 \%$. MW $=795.71$. Anal. Calcd. for $\mathrm{C}_{39} \mathrm{H}_{32} \mathrm{~N}_{6} \mathrm{O}_{8} \mathrm{SV}: \mathrm{C}, 58.87 ; \mathrm{H}, 4.05 ; \mathrm{N}$, 10.56. Found: $\mathrm{C}, 59.35 ; \mathrm{H}, 4.55 ; \mathrm{N}, 10.75 \%$. The complex is paramagnetic, $\mu_{\text {eff }}=0.93 \mu_{\mathrm{B}}$. FT-IR $\left(\mathrm{KBr}, \mathrm{cm}^{-1}\right): 3050(\mathrm{w})$, 1586 (s), 1554 (s), 1501 (vs), 1423 (s), 1400 (s), 1369 (w), 1345 (w), 1318 (w), 1288 (w), 1217 (w), 1173 (w), 1143 (w), 1105 (w), 1073 (w), 1025 (m), 956 (vs), 846 (s), 770 (w), $722(\mathrm{vs}), 703(\mathrm{w}), 654(\mathrm{w}), 608(\mathrm{w}), 522(\mathrm{w}), 469(\mathrm{w})$,
423 (w). UV-Vis (DMSO): $367 \mathrm{~nm}(\mathrm{MeCN}): 271,387 \mathrm{~nm}$, (EtOH): 226, 266, $377 \mathrm{~nm}$.

\section{Preparation of $\left[\mathrm{VO}\left(\mathrm{HL}_{17}\right)\left(\mathrm{H}_{2} \mathrm{O}\right)\right] \cdot 2 \mathrm{H}_{2} \mathrm{O}(23)$}

Potassium salt of 5-sulfosalicylaldehyde ( $480 \mathrm{mg}, 2.0 \mathrm{mmol}$ ) and nicotinic acid hydrazide $(274 \mathrm{mg}, 2.0 \mathrm{mmol})$ in $100 \mathrm{ml}$ ethanol were refluxed for $20 \mathrm{~min}$ under Ar. Then, [VO(acac $\left.)_{2}\right](530 \mathrm{mg}, 2.0 \mathrm{mmol})$ was added. The reflux was continued for $45 \mathrm{~min}$ under Ar. The obtained compound was filtered off, washed several times with ethanol, and dried at room temperature. Yield: $0.637 \mathrm{~g}, 67 \%$. $\mathrm{MW}=479.37$. Anal. Calcd. for $\mathrm{C}_{13} \mathrm{H}_{15} \mathrm{~N}_{3} \mathrm{O}_{9} \mathrm{KSV}$ : C, 32.54; H, 3.13; N, 8.76; S, $6.70 \%$. Found: C, 33.04; H, 2.77; N, 8.44; S, 6.66\%. The complex is paramagnetic, $\mu_{\mathrm{eff}}=1.19 \mu_{\mathrm{B}}$. FT-IR $\left(\mathrm{KBr}, \mathrm{cm}^{-1}\right)$ : 3421 (vs), 1613 (vs), 1534 (m), 1461 (m), 1414 (w), 1384 (m), 1363 (m), 1298 (w), 1193 (s), 1113 (s), 1035 (vs), 912 (m), $883(\mathrm{~m}), 832(\mathrm{w}), 720(\mathrm{w}), 671(\mathrm{w}), 619(\mathrm{~s}), 535(\mathrm{w})$, 458 (w), 415 (w). UV-Vis (DMSO): 326, $409 \mathrm{~nm},(\mathrm{MeCN})$ : 242, 325, $405 \mathrm{~nm}$, (EtOH): 320, $399 \mathrm{~nm}$.

\section{Preparation of $\mathrm{K}_{4}\left[\left\{\mathrm{VO}\left(\mathrm{L}_{18}\right)\right\}_{2}\left(\mathrm{SO}_{4}\right)\right] \cdot 2 \mathrm{H}_{2} \mathrm{O} \cdot \mathrm{EtOH}(24)$}

Potassium salt of 5-sulfosalicylaldehyde ( $361 \mathrm{mg}, 1.5 \mathrm{mmol}$ ) and 3-hydroxy-2-naphtoic acid hydrazide $(303 \mathrm{mg}$, $1.5 \mathrm{mmol}$ ) were dissolved in $60 \mathrm{ml}$ ethanol under Ar flow on a magnetic stirrer. The mixture was refluxed for $20 \mathrm{~min}$ with constant stirring, and the temperature was raised to $100{ }^{\circ} \mathrm{C}$. Then, $\mathrm{VOSO}_{4}(333 \mathrm{mg}, 1.5 \mathrm{mmol})$ in $15 \mathrm{ml}$ ethanol was added. The reflux was continued for 180 min under Ar. After that, the volume of the reaction mixture was concentrated to final volume of ca. $20 \mathrm{ml}$ and cooled to room temperature. The obtained compound was filtered off, washed several times with ethanol, and dried in air. Yield: $0.766 \mathrm{~g}$, 85\%. $\mathrm{MW}=1197.06$. Anal. Calcd. for $\mathrm{C}_{38} \mathrm{H}_{32} \mathrm{~K}_{4} \mathrm{~N}_{4} \mathrm{O}_{21} \mathrm{~S}_{3} \mathrm{~V}_{2}$ : C, 37.01; H, 2.61; N, 4.54; S, 7.79\%. Found: C, 37.35; H, 2.67; N, 4.66; S, 8.54\%. The complex is diamagnetic. FT-IR $\left(\mathrm{KBr}, \mathrm{cm}^{-1}\right)$ : 3471 (w, br), $3290(\mathrm{w}), 3081(\mathrm{w}), 1629(\mathrm{~m})$, 1600 (s), 1578 (w), 1540 (vs), 1462 (w), 1375 (s), 1229 (s), 1138 (vs), 1106 (vs), 1039 (s), 977 (s), 918 (w), 886 (vs), 835 (w), $761(\mathrm{w}), 727$ (w), 617 (s), $583(\mathrm{w}), 505(\mathrm{w}), 454$ (w), 429 (w). UV-Vis (DMSO): 324, $414 \mathrm{~nm}$, (EtOH): 225, $274,326 \mathrm{~nm}$.

\section{Crystallographic data collection and structure refinement}

Diffraction intensity data for a single crystal of three new compounds were collected at $293 \mathrm{~K}$ on an Oxford Diffraction Super Nova diffractometer using monochromatic Mo $\mathrm{K}_{\alpha}(\mathbf{1 0}, \mathbf{1 6})$ or $\mathrm{Cu} \mathrm{K}_{\alpha}(\mathbf{4})$ radiation at $\lambda=0.71073 \AA$ and $\lambda=1.5418 \AA$, respectively. Cell refinement and data reduction were performed using firmware [34]. Positions of all 
nonhydrogen atoms were determined by direct methods using SIR software (4, 10: SIR92 [35], 16: SIR97 [36]. All nonhydrogen atoms were refined anisotropically using weighted full-matrix least-squares on $F^{2}$. Refinement and further calculations were carried out using SHELXL software [37] (4, 10: ver 2017/1, 16: ver 2016/1]. All hydrogen atoms joined to carbon atoms were positioned with idealized geometries and refined using a riding model with $U_{\text {iso }}(\mathrm{H})$ fixed at $1.2 U_{\text {eq }}\left(\mathrm{C}_{\text {arom }}\right)$. In structure $\mathbf{1 0}$, two carbon atoms of the coordinating ethanol molecule, C31 (methylene) and C32 (methyl), show disorder and were modeled (split) into two sets of positions A and B with their geometries restrained, with the refined occupancies being 0.54(5) and $0.46(5)$, respectively. The positions of all hydrogen atoms were constrained for all compounds using AFIX (SHELXL) commands. CCDC 1871461, 1871462, and 1871642 contain the supplementary crystallographic data for 4,10 , and 16 . These data can be obtained free of charge from The Cambridge Crystallographic Data Center via www.ccdc.cam. ac.uk/data_request/cif. The crystallographic data and detailed information on the structure solution and refinement are presented in Table 2. The bond distances and angles are listed in Supplementary Tables S1-S3.

\section{Results and discussion}

\section{General remarks on the synthesis}

As a result of the syntheses, 24 oxidovanadium(IV,V) complexes were obtained by reaction of $\mathrm{VOSO}_{4}$ aq or $\left[\mathrm{VO}(\mathrm{acac})_{2}\right]$ with in situ formed tridentate Schiff base

Table 2 Crystal data and structure refinement parameters for 4, 10, and 16

\begin{tabular}{|c|c|c|c|}
\hline & 4 & 10 & 16 \\
\hline Empirical formula & $\mathrm{C}_{16} \mathrm{H}_{14} \mathrm{~N}_{3} \mathrm{O}_{7} \mathrm{~V}$ & $\mathrm{C}_{22} \mathrm{H}_{22} \mathrm{~N}_{3} \mathrm{O}_{8} \mathrm{~V}$ & $\mathrm{C}_{20} \mathrm{H}_{25} \mathrm{~N}_{2} \mathrm{O}_{6} \mathrm{~V}$ \\
\hline Formula weight & 411.24 & 507.36 & 440.36 \\
\hline Temperature (K) & 293(2) & 293(2) & $293(2)$ \\
\hline Wavelength $(\AA)$ & 1.5418 & 0.71069 & 0.71073 \\
\hline Crystal system & Triclinic & Monoclinic & Triclinic \\
\hline Space group & $P-1$ & $P 21 / c$ & $P-1$ \\
\hline \multicolumn{4}{|l|}{ Unit cell dimensions } \\
\hline$a(\AA)$ & $7.4393(4)$ & $11.163(5)$ & $10.467(5)$ \\
\hline$b(\AA)$ & $8.6931(5)$ & $28.248(5)$ & $10.683(5)$ \\
\hline$c(\AA)$ & $13.2880(8)$ & $7.563(5)$ & $10.991(5)$ \\
\hline$\alpha\left(^{\circ}\right)$ & $88.914(5)$ & 90 & $118.752(5)$ \\
\hline$\beta\left({ }^{\circ}\right)$ & $80.414(5)$ & $94.337(5)$ & $94.900(5)$ \\
\hline$\gamma\left({ }^{\circ}\right)$ & $82.214(5)$ & 90 & $96.272(5)$ \\
\hline Volume $\left(\AA^{3}\right)$ & $839.53(8)$ & $2378.0(19)$ & $1057.5(9)$ \\
\hline$Z$ & 2 & 4 & 2 \\
\hline Density, calculated $\left(\mathrm{Mg} / \mathrm{m}^{3}\right)$ & 1.627 & 1.417 & 1.383 \\
\hline Absorption coefficient $\left(\mathrm{mm}^{-1}\right)$ & 5.383 & 0.469 & 0.507 \\
\hline$F(000)$ & 420 & 1048 & 460 \\
\hline Crystal size $\left(\mathrm{mm}^{3}\right)$ & $0.260 \times 0.120 \times 0.060$ & $0.460 \times 0.340 \times 0.140$ & $0.630 \times 0.190 \times 0.100$ \\
\hline$\theta$ range for data collection $\left(^{\circ}\right)$ & 3.373 to 61.167 & 3.416 to 26.367 & 3.722 to 26.374 \\
\hline Index ranges & $-8 \leq h \leq 8,-9 \leq k \leq 9,-3 \leq l \leq 15$ & $\begin{aligned}-13 & \leq h \leq 13,-35 \leq k \leq 35 \\
-7 & \leq l \leq 9\end{aligned}$ & $\begin{array}{l}-13 \leq h \leq 13,-13 \leq k \leq 13 \\
-13 \leq l \leq 13\end{array}$ \\
\hline Reflections collected & 2545 & 15,774 & 13,522 \\
\hline Independent reflections & $2545[R($ int $)=0.0457]$ & $4834[R($ int $)=0.0207]$ & $4316[R($ int $)=0.0224]$ \\
\hline Completeness to $\theta(\%)$ & 98.8 & 99.4 & 99.7 \\
\hline Absorption correction & Multiscan & Multiscan & Multiscan \\
\hline Refinement method & Full-matrix least-squares on $F^{2}$ & Full-matrix least-squares on $F^{2}$ & Full-matrix least-squares on $F^{2}$ \\
\hline Data/restraints/parameters & $2545 / 14 / 250$ & $4834 / 25 / 332$ & $4316 / 0 / 269$ \\
\hline Goodness-of-fit on $F^{2}$ & 1.163 & 1.087 & 1.082 \\
\hline Final $R$ indices $[I>2 \sigma(I)]$ & $R_{1}=0.0682, w R_{2}=0.1903$ & $R_{1}=0.0433, w R_{2}=0.1374$ & $R_{1}=0.0356, w R_{2}=0.1010$ \\
\hline$R$ indices (all data) & $R_{1}=0.0795, w R_{2}=0.1946$ & $R_{1}=0.0577, w R_{2}=0.1437$ & $R_{1}=0.0469, w R_{2}=0.1059$ \\
\hline Largest diff. peak and hole $\left(\mathrm{e} / \AA^{3}\right)$ & 0.893 and -0.477 & 0.707 and -0.247 & 0.329 and -0.322 \\
\hline
\end{tabular}


ligands. Six of these $(4,7,10,16,19$, and 24$)$ are $V(V)$ complexes, while the others are V(IV) complexes. In some syntheses, co-ligand in the form of 2,2'-bipyridine or 1,10'-phenanthroline was used to precipitate the compounds. As vanadium source, $\mathrm{VOSO}_{4}$ aq or $\left[\mathrm{VO}(\mathrm{acac})_{2}\right]$ was used. In general, $\left[\mathrm{VO}(\mathrm{acac})_{2}\right]$ was found to be more suitable due to solubility; however, the product composition in most cases was similar, independently of the complex used. Only in three cases $(\mathbf{1}, \mathbf{1 2}$, and 24) was $\mathrm{SO}_{4}^{2-}$ group noticed to be present in the complex and could not be removed by simple washing. The analysis indicates that one ion per two vanadium centers is present, in all the performed syntheses. Thus, the dimeric form of the complex is suggested.

Usually, in our previous investigations with vanadium complexes based on Schiff base ligands, oxidation of V(IV) to $\mathrm{V}(\mathrm{V})$ when co-ligand (for example, phen) was not used $[22,23,25,27,28]$ was observed. In this part of the investigation, the oxidovanadium(IV) complexes $(\mathbf{1}, \mathbf{9}, \mathbf{1 1}, \mathbf{1 2}$, 18,23 ) were obtained, among others, for which there was no change in the oxidation state and for which a stabilizing ligand for the V(IV) oxidation state was not used in the synthesis. It must be underlined, however, that those complexes are not as stable in time as those with co-ligands, especially with phen.

As the syntheses were performed under Ar atmosphere, it was difficult to dry the samples to constant mass due to their air sensitivity. Moreover, the samples were weighed in air for elemental analysis. This caused the analyses not to fit perfectly (within $0.4 \%$ ) in several cases to a proposed formula (in two cases the largest mistake was on hydrogen, but still less than $0.9 \%$ ).

\section{Description of the structures}

Compound 4 crystallizes in the triclinic space group $P$ - $l$ with an asymmetric cell unit (Fig. 1) containing the monomeric complex of $\left[\mathrm{VO}\left(\mathrm{L}_{2}\right)(\mathrm{EtO})\right]$. The central vanadium atom has a five-coordinate environment with VO4N composition, taking the shape of an almost perfect square pyramid. The square base of the polyhedron is made of the atoms of a tridentate L2 ligand and consists of phenolic oxygen O8, imine nitrogen $\mathrm{N} 4$, enol oxygen $\mathrm{O} 1$, and an oxygen atom $\mathrm{O} 24$ from deprotonated ethoxide oxygen. The apical position of the square pyramid is occupied by the terminal oxido $\left(\mathrm{O}^{2-}\right)$ group O23. The short V1-O23 distance of 1.566(5) $\AA$ indicates the presence of a double vanadium-oxygen bond $(\mathrm{V}=\mathrm{O})$. The central vanadium $\mathrm{V} 1$ atom is located above the basal plane of O24, O1, N4, and O8 by $0.450 \AA$ and is directed toward the apical oxido ligand. Both L2 aromatic rings are slightly bent downwards in relation to the plane they create, and the highest deviation relates to the $\mathrm{C} 17$ and C11 atoms and amounts to $0.341 \AA$ and $0.233 \AA$, respectively (Fig. 1). The square pyramid is slightly distorted, in which the individual angles $\mathrm{N} 4-\mathrm{O} 1-\mathrm{O} 24, \mathrm{O} 1-\mathrm{O} 24-\mathrm{O} 8$, $\mathrm{O} 24-\mathrm{O} 8-\mathrm{N} 4$, and $\mathrm{O} 8-\mathrm{N} 4-\mathrm{O} 1$ are $97.16^{\circ}, 85.10^{\circ}, 87.69^{\circ}$, and $89.96^{\circ}$, respectively, where the highest deviation from the ideal $90^{\circ}$ is $7.16^{\circ}$. The observed distortion is due to the intramolecular structural tension caused by the presence of both five- and six-membered rings in the square plane of the pyramid. Packing analysis of the crystal structure reveals that the complex molecules are held together by two $\pi \ldots \pi$ stacking and four weak intermolecular interactions of $\mathrm{C}-\mathrm{H}$...O type, as summarized in Supplementary Tables S4 and S5.

The structure of $\mathbf{1 0}$ with atom numbering scheme and coordination polyhedron of vanadium is presented in Fig. 2. The vanadium atom is surrounded by $\mathrm{ONO}$ donor atoms of ligand $\mathrm{L}$, two oxygens from $\mathrm{EtOH}$ and $\mathrm{EtO}^{-}$molecules, and $\mathrm{O}^{2-}$ anion. The central vanadium atom has six-coordinated surrounding with ethoxy and L ligand in plane and $\mathrm{EtOH}$ and $\mathrm{O}^{2-}$ as apexes. The vanadium atom is shifted out of plane (constructed on N15-O13-O27 atoms) toward the oxygen atom by $0.286 \AA$. Contrary to $\mathbf{4}$, in $\mathbf{1 0}$ aromatic rings are shifted below this plane (hydrazide ones) and above the plane (from aldehyde moieties) with the longest distances to plane for C7 (0.676 $\AA$ ) and C21 (0.387 $\mathrm{A})$. The octahedron is slightly distorted, with $\mathrm{O} 33-\mathrm{V} 1-\mathrm{O} 30$ angle of $174.28^{\circ}$.
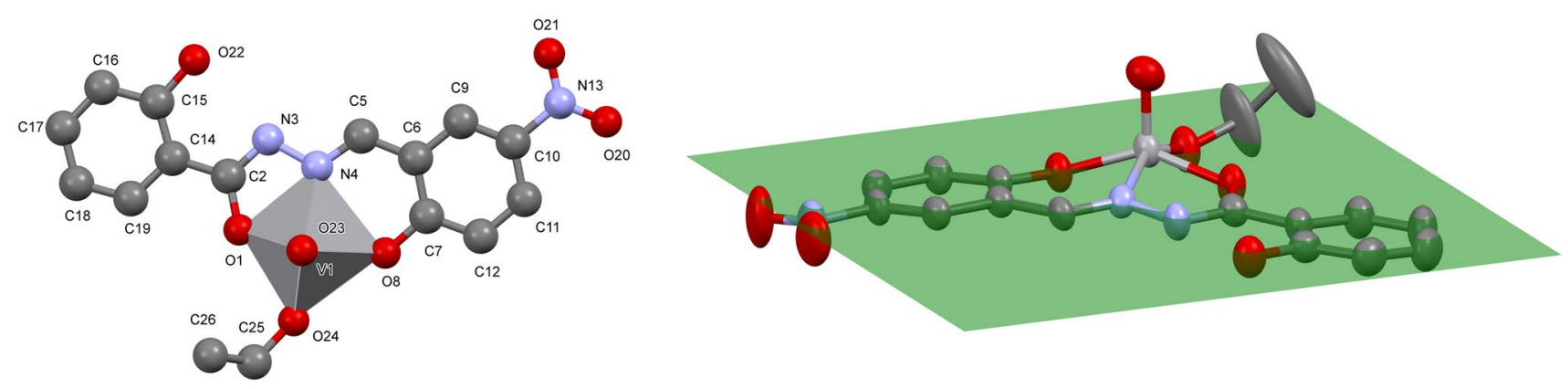

Fig. 1 Structure of $\mathbf{4}$ with marked coordination polyhedron and atom labeling scheme (left), showing the planarity of the ligand and donor atoms with plane constructed on N4-O1-O24 atoms with 30\% probability ellipsoids (right). Hydrogen atoms omitted for clarity 

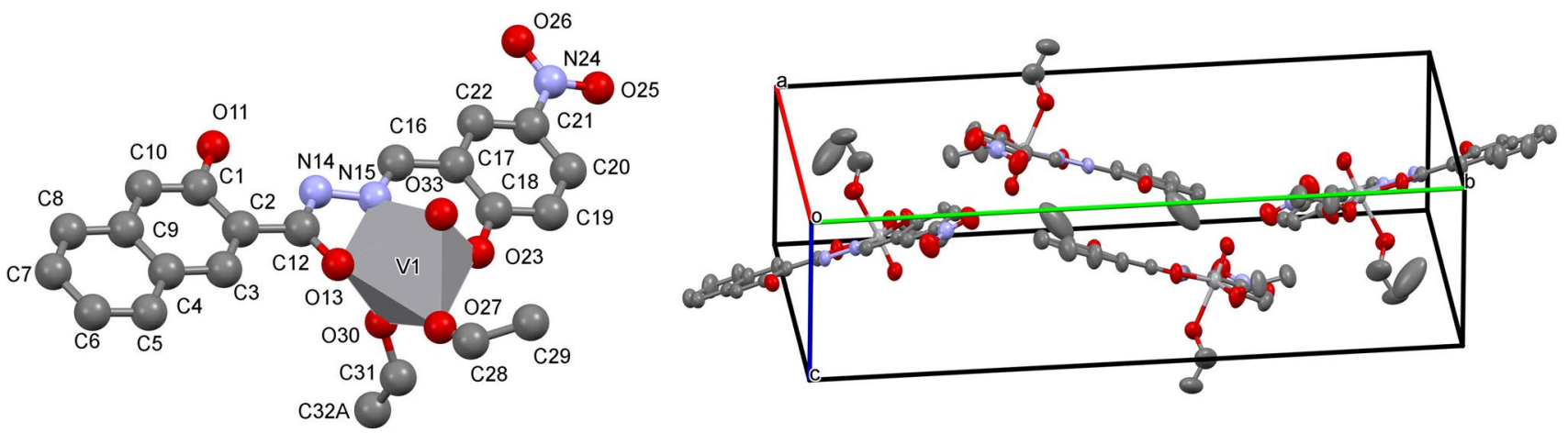

Fig. 2 Structure of $\mathbf{1 0}$ with marked coordination polyhedron and atom labeling scheme. The packing in $\mathbf{1 0}$ with $30 \%$ probability ellipsoids (right). Hydrogen atoms omitted for clarity

Again, as in 4, distortion of the square-pyramidal plane N15-O13-O27-O23, caused by the presence of five- and six-membered rings, is observed.

Similarly to $\mathbf{4}$, in the cell unit there is an equimolar mixture of two isomers, but in the case of $\mathbf{1 0}$, four molecules are present in the unit cell. The packing is presented in Fig. 2 (right).

In 10, both intra- and intermolecular hydrogen bonds are present. The intramolecular one is between N14 and O11, similarly to 4 , with a distance of $2.577(2) \AA$, slightly shorter than in 4. The intermolecular hydrogen bond is formed between O11 and O30, with a distance equal to 2.784(2) $\AA$ (Supplementary Table S6).

The structure of $\mathbf{1 0}$ consists of separated layers, as presented in Fig. 3. While the layers are supported by a net of hydrogen bonds, only very weak intramolecular interactions are present between layers.

The structure of the asymmetric parts of the unit cell of complex 16 and the adopted atomic numbering scheme are shown in Fig. 4. This complex crystallizes in the triclinic space group $P-l$ with the asymmetric unit cell containing one molecule of $\left[\mathrm{VO}\left(\mathrm{L}_{11}\right)(\mathrm{EtO})(\mathrm{EtOH})\right]$ complex. Oxidovanadium cation $\left(\mathrm{VO}^{2+}\right)$ is coordinated with the fully deprotonated tridentate hydrazone ligand $\left(\mathrm{L}_{11}\right)$ through phenolate oxygen $(\mathrm{O} 7)$, azomethine nitrogen (N11), and enolate oxygen $(\mathrm{O} 21)$ and additionally with two oxygen atoms, one of which is deprotonated ethoxide oxygen $(\mathrm{O} 22)$, while the second oxygen $(\mathrm{O} 25)$ comes from the ethanol molecule. Thus, the coordination environment of the central atom $\mathrm{V}$ adopts the geometry of an elongated octahedron with the scheme $\mathrm{O} 5 \mathrm{~N}$. The basic plane is defined by the atoms $\mathrm{O} 7, \mathrm{O} 21, \mathrm{O} 22$, and $\mathrm{N} 11$, while the central vanadium atom is above this plane by $0.3037(8)$ $\AA$ and is shifted toward the $\mathrm{O} 28$ atom (the $\mathrm{V}-\mathrm{O}$ distance equals $1.583(2) \AA$ ). In the discussed complex (16), the lengths of $\mathrm{V}-\mathrm{O}$ bonds have different values, the $\mathrm{V}=\mathrm{O}_{\text {oxido }}$ bond being the shortest and the $\mathrm{V}-\mathrm{O}_{\text {enolate }}=2.383(2) \AA$ (O25 of ethanol) bond being the longest. The oxygen $(\mathrm{O} 28)$ atom and atom $\mathrm{O} 25$ of the ethanol molecule occupy the apical sites of the octahedron with a trans angle of

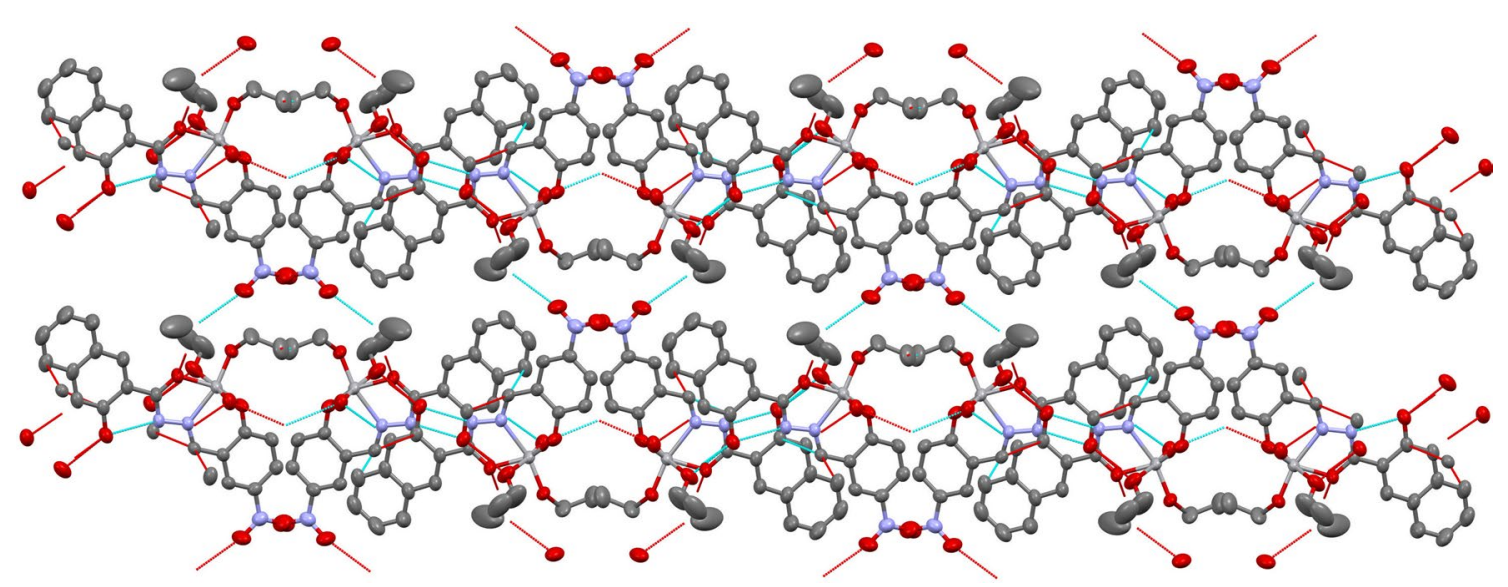

Fig. 3 Packing scheme in 10; the net of hydrogen bonds and short contacts are visualized. Hydrogen atoms omitted for clarity, with $30 \%$ probability ellipsoids shown 

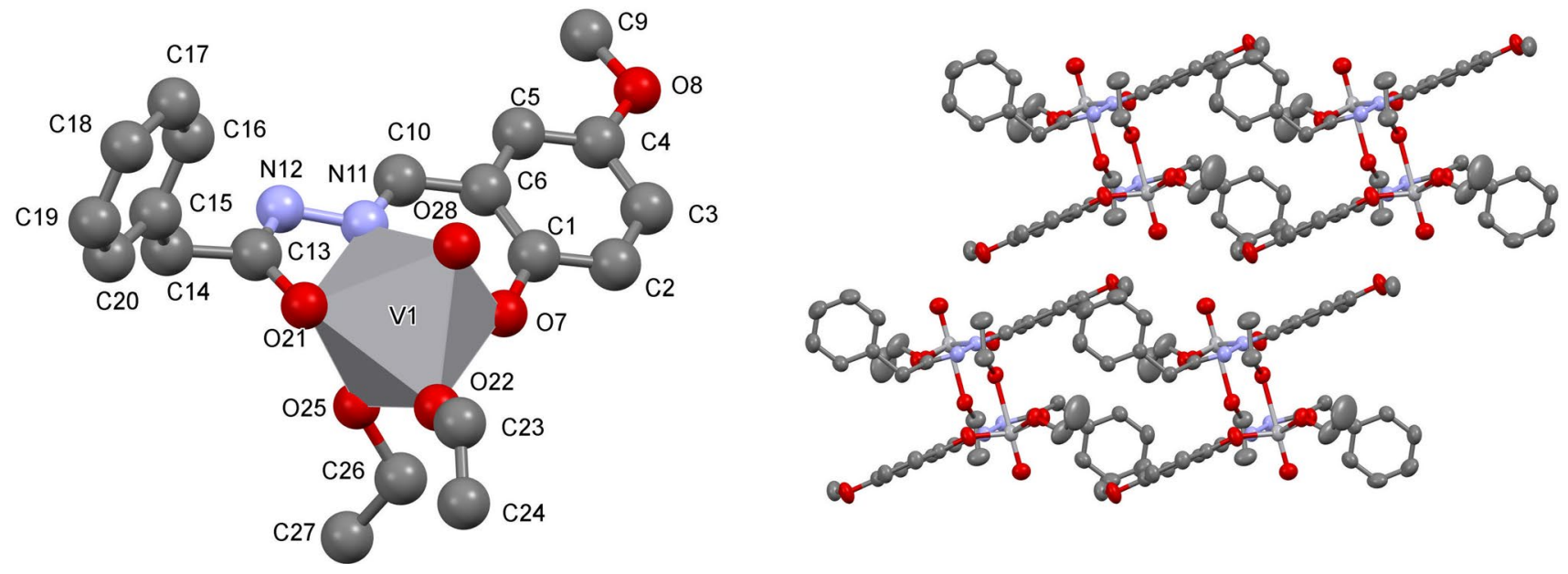

Fig. 4 Structure of $\mathbf{1 6}$ with marked coordination polyhedron and atom labeling scheme (left). Packing scheme in 16, with $30 \%$ probability ellipsoids (right). Hydrogen atoms omitted for clarity

$174.7(6)^{\circ}$. The phenyl ring (C15-C20) of the ligand forms a dihedral angle of $112.6(3)^{\circ}$ with the 2-hydroxy5-methoxyphenyl ring. This causes intracomplex tension and, consequently, is an additional cause of the octahedral deformation of the geometry of the coordination environment of the central vanadium atom. Packing analysis of the crystal structure of $\mathbf{1 6}$ reveals that the complex molecules are stabilized by one typical hydrogen interaction of $\mathrm{O}-\mathrm{H} \ldots \mathrm{N}$ type $[\mathrm{O}(25)-\mathrm{H}(25) \ldots \mathrm{N}(12)[-x+1,-y+1$, $-z+1], d(\mathrm{D}-\mathrm{H})=0.76(2) \AA, d(\mathrm{H} \ldots \mathrm{A})=2.07(2) \AA$, $d(\mathrm{D} \ldots \mathrm{A})=2.823(2) \AA$, and $\left.\angle(\mathrm{DHA})=172(2)^{\circ}\right]$ (Supplementary Table S7). The long-distance packing scheme is presented in Fig. 4 (right).

A comparison of the $\mathrm{V}=\mathrm{O}$ distances in 4, 10, and 16, of $1.568,1.582$, and $1.583 \AA$, respectively, reflects the presence of EtOH ligand in $\mathbf{1 0}$ and 16, resulting in an increase in the bond distance by ca. $0.014 \AA$ compared with 4 . The $\mathrm{V}=\mathrm{O}$ bond distance in 4, 10, and 16 is shorter than that observed in V(IV) analogs $(1.604,1.606,1.593,1.598$, and $1.594 \AA$ ), and similar shortening is observed also for the $\mathrm{V}-\mathrm{O}_{\mathrm{L}}(\mathrm{L}=$ Schiff base ligand $)$ bond $[21,22]$. In contrast, the $\mathrm{V}-\mathrm{N}_{\mathrm{L}}$ distance is longer in $\mathrm{V}(\mathrm{V})$ compared with $\mathrm{V}(\mathrm{IV})$ complexes (bond distances 2.046, 2.058, 2.039, 2.038, and $2.031 \AA$ ) $[21,22]$.

\section{IR spectra}

IR spectral measurements confirmed the presence of characteristic groups in the synthesized oxidovanadium complexes for both V(IV) and V(V). All the vibrational bands are listed in the "Experimental" section. The most characteristic strong band located in the range of 955-965 $\mathrm{cm}^{-1}$ is associated with $\nu(\mathrm{V}=\mathrm{O})$ vibration in $\mathrm{V}(\mathrm{IV})$ complexes.
This is a direct difference from the $\mathrm{V}(\mathrm{V})$ complexes, where weak bands in this range or intense bands at $983 \mathrm{~cm}^{-1}$ are observed. The investigated complexes also show a band at ca. $1600 \mathrm{~cm}^{-1}$ that can be assigned to $\nu(\mathrm{C}=\mathrm{N})$ vibration in a Schiff base ligand. For complexes with phen or bpy as ligand, the characteristic bands at ca. 1490, 1425, and $725 \mathrm{~cm}^{-1}$ are related to the vibration in these ligands and were also observed in other $d$-electron metal complexes with phen or bpy [38].

\section{UV-Vis spectra}

The positions of the bands for the compounds in three selected solvents (DMSO, MeCN, and EtOH) were presented above. The UV-Vis spectra for all the complexes in organic solvents [DMF, DMSO, EtOH, $\mathrm{MeOH}, \mathrm{MeCN}$, dichloroethane $\left(\mathrm{EtCl}_{2}\right)$, and $n$-octanol] and in solid state as well as their stability at neutral pH and at $\mathrm{pH} 2.00$ are presented in the Supplementary Electronic Material. In the UV part of the electronic spectra, the bands attributed to the coordinated Schiff base ligand and co-ligand (phen or bpy) where present are observed as separate peaks. In the visible part of the spectra, the band located at ca. $400 \mathrm{~nm}$ can be assigned to a ligand-to-metal charge-transfer (LMCT) transition between the metal center and Schiff base ligand. This band is relatively intense with molar extinction coefficient $\left(\mathrm{mol}^{-1} \mathrm{~cm}^{-1} \mathrm{dm}^{3}\right)$ in DMSO of $2.56 \times 10^{4}(\mathbf{1}, 390 \mathrm{~nm})$, $2.89 \times 10^{4}(2,393 \mathrm{~nm}), 2,61 \times 10^{4}(3,399 \mathrm{~nm}), 4.97 \times 10^{4}$ $(\mathbf{4}, 387 \mathrm{~nm})$, and $2.55 \times 10^{4}(\mathbf{5}, 390 \mathrm{~nm})$.

The reflectance spectra of representative complexes [oxidovanadium(IV) complexes with bpy and phen as coligand and oxidovanadium(V) complex] are presented in Fig. 5. Complexes 2 and $\mathbf{3}$ show absorption above $700 \mathrm{~nm}$, which is typical for $d^{1}$ configuration of $\mathrm{V}(\mathrm{IV})$ and is in 


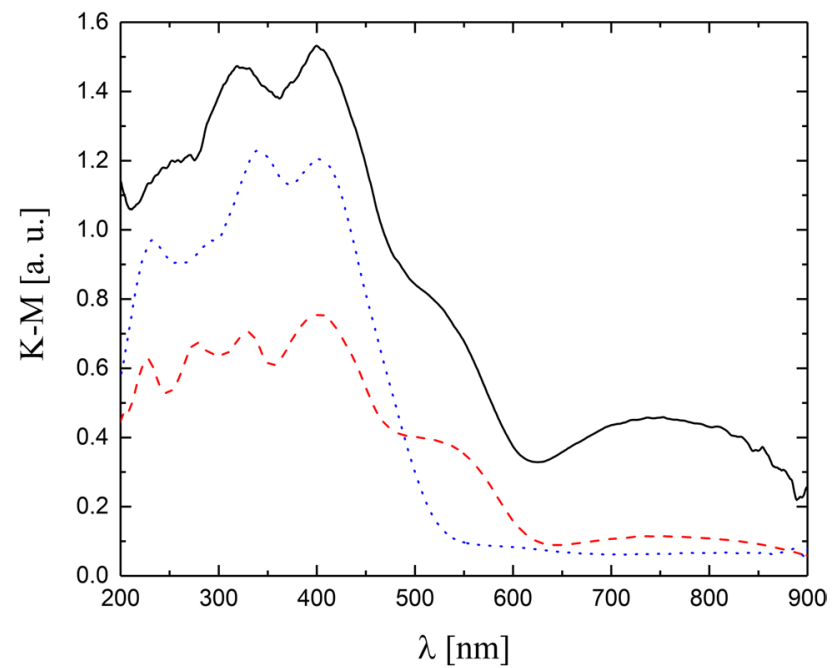

Fig. 5 Reflectance spectra of complexes 2 (black, solid line), 3 (red, dashed line), and $\mathbf{1 0}$ (blue, dotted line) in the range 200-900 $\mathrm{nm}$ after Kubelka-Munk transformation. $\mathrm{BaSO}_{4}$ as internal (white) reference
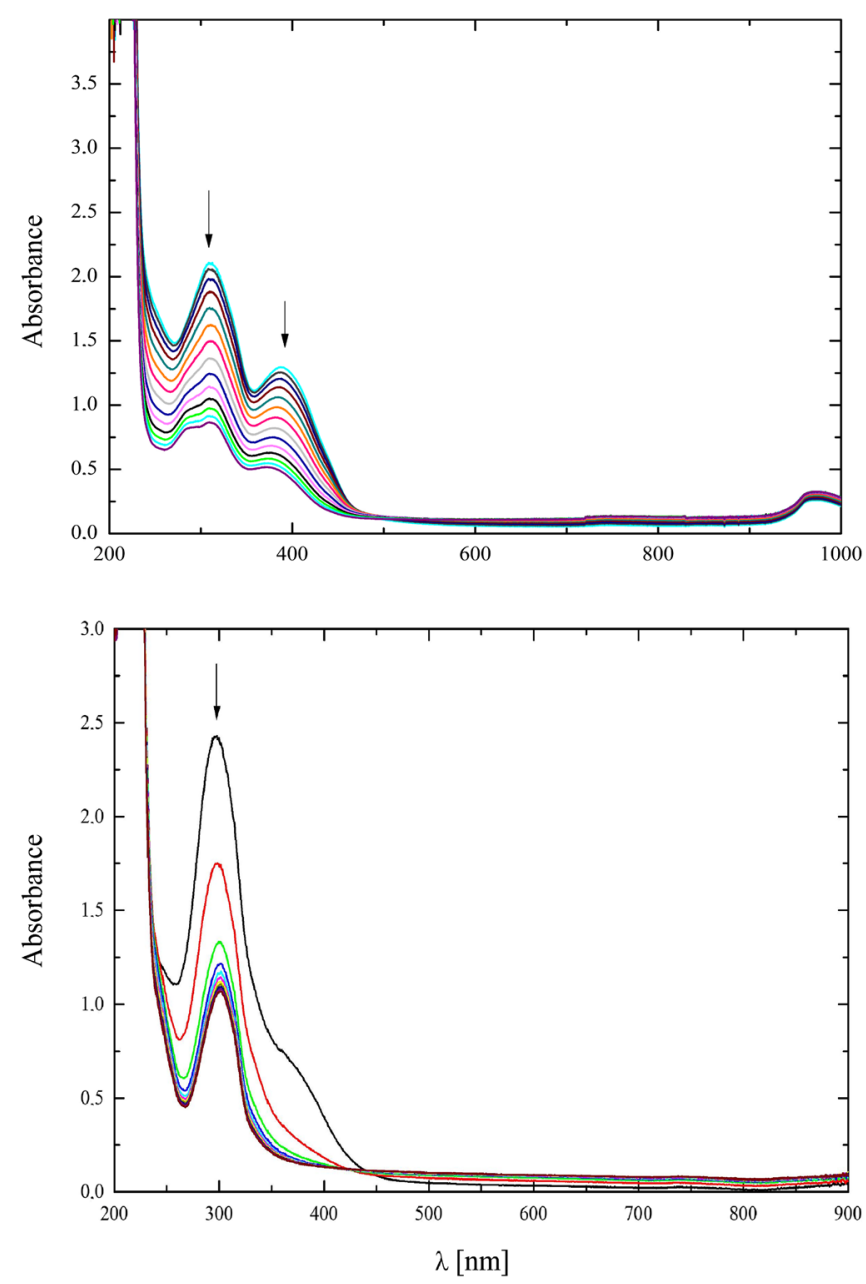

accordance with the magnetic measurements presented above. Due to low solubility of all complexes, the $d-d$ transition can be observed only in reflectance spectra, while in solution it is obscured by low intensity. For 10, no band appears in the range above $500 \mathrm{~nm}$, confirming the $\mathrm{V}(\mathrm{V})$ oxidation state and $d^{0}$ configuration. This situation was also observed for the other $\mathrm{V}(\mathrm{V})$ complexes $(4,7,10,16,19$, and 24).

Due to problems with the insolubility of the obtained compounds in water, the complexes were studied in DMSO. This solvent was selected because of its usefulness in, e.g., medicine, as it supports transfer of substances through biological membranes. The stability of the selected complexes was measured by UV-Vis spectra versus time. The compounds showed stability in the solvent tested and were dissolved in DMSO- $\mathrm{H}_{2} \mathrm{O}$ mixture $(20 \mu \mathrm{l}+3 \mathrm{ml}$, respectively) at neutral $\mathrm{pH}$ and at $\mathrm{pH}$ 2.00. The UV-Vis spectra were measured over time for each mixture. Most of the complexes tested were stable in natural $\mathrm{pH}$ but gradually decomposed in
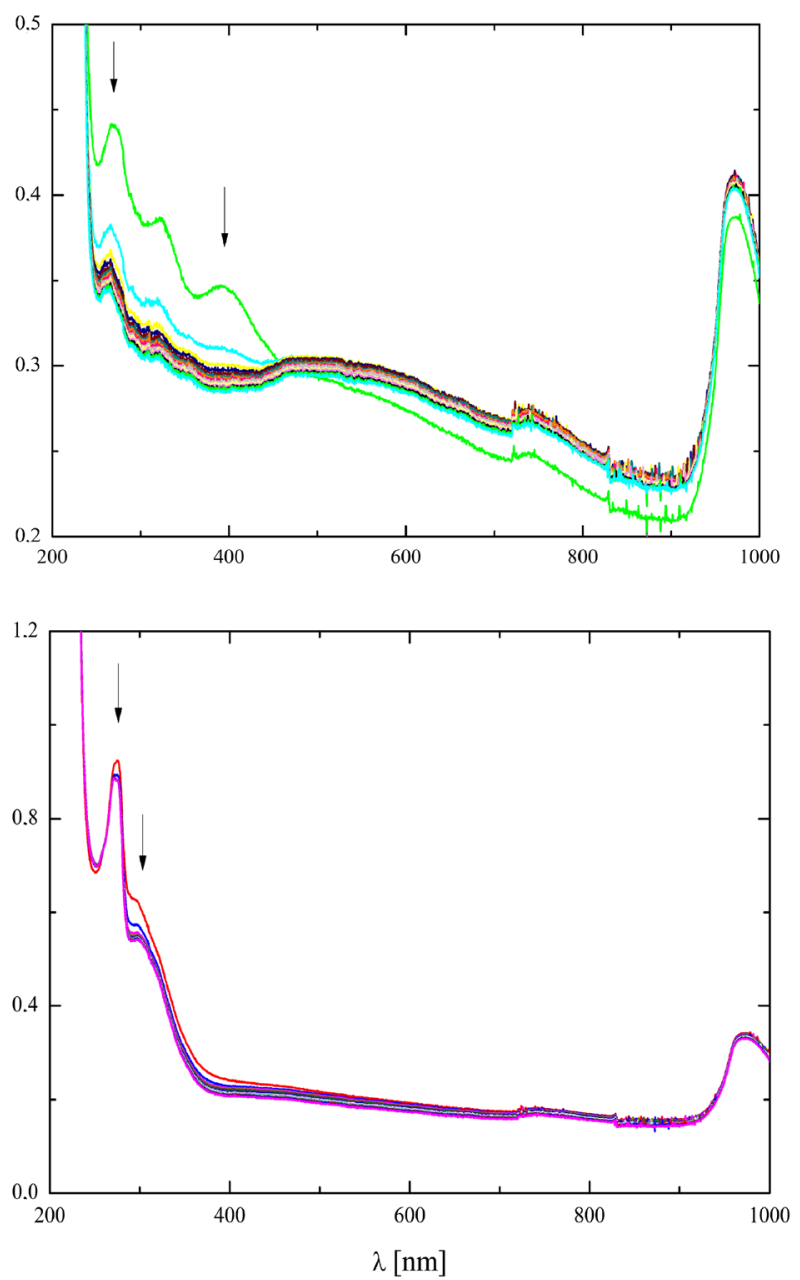

Fig. $6 \mathrm{UV}-$ Vis spectra of complex 2 (left side) and 3 (right side) in DMSO- $\mathrm{H}_{2} \mathrm{O}$ mixture $(20 \mu \mathrm{l}+3 \mathrm{ml}$, respectively) at natural $\mathrm{pH}$ (above) and pH 2.00 (below). $T=37^{\circ} \mathrm{C}, d=1 \mathrm{~cm}, 15$ spectra measured at 340 -s intervals. The arrow shows the direction of changes in spectra 


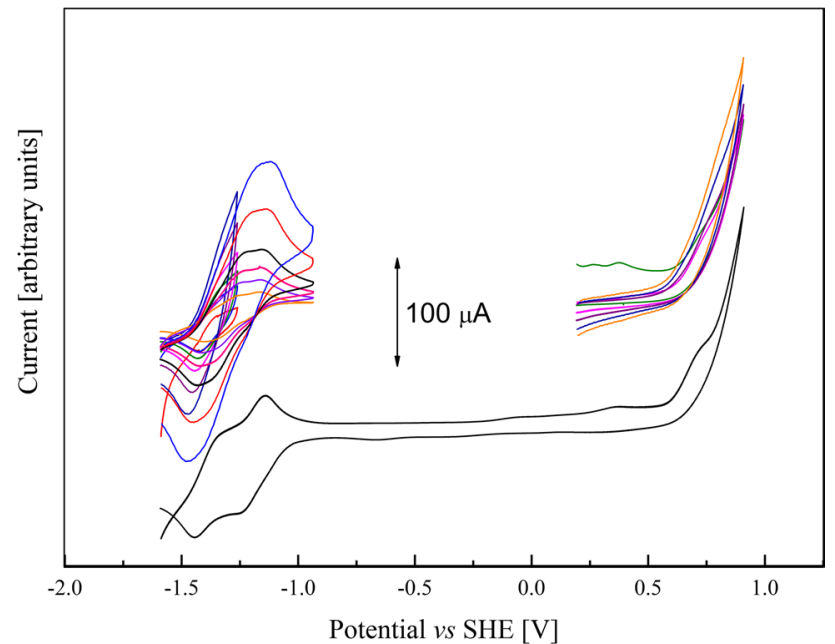

Fig. 7 Cyclic voltammogram of $\mathbf{3}$ in DMSO. 0.1 $\mathrm{M} \mathrm{Bu}_{4} \mathrm{NPF}_{6}$ as electrolyte, Pt working and counter and $\mathrm{Ag} / \mathrm{AgCl}$ reference electrodes, potentials versus NHE. Scan speed $100 \mathrm{mV} / \mathrm{s}$ (for main curve in full range); insets: scans in selected voltage range with scan speeds in the range of $20-1000 \mathrm{mV} / \mathrm{s}$

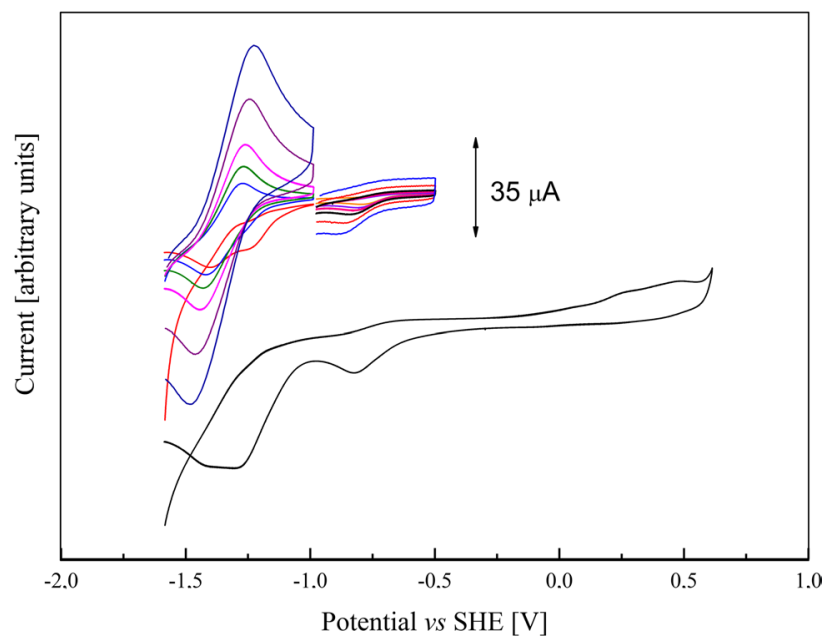

Fig. 8 Cyclic voltammogram of 5 in DMSO. 0.1 $\mathrm{M} \mathrm{Bu}_{4} \mathrm{NPF}_{6}$ as electrolyte, Pt working and counter and $\mathrm{Ag} / \mathrm{AgCl}$ reference electrodes, potentials versus NHE. Scan speed $100 \mathrm{mV} / \mathrm{s}$ (for main curve in full range); insets: scans in selected voltage range with scan speeds in the range of $20-1000 \mathrm{mV} / \mathrm{s}$

pH $2.00(\mathbf{1}, \mathbf{3}, \mathbf{6}, \mathbf{8}-\mathbf{1 0}, \mathbf{1 2}-\mathbf{1 5}, \mathbf{1 8}-\mathbf{2 1}, \mathbf{2 3}, \mathbf{2 4})$. Complexes $\mathbf{2}$, 4,17 , and 22 were unstable at natural $\mathrm{pH}$ and $\mathrm{pH} 2.00$. Only complexes $\mathbf{7}$ and $\mathbf{1 6}$ were stable in natural $\mathrm{pH}$ as well as $\mathrm{pH}$ 2.00. Exemplary spectral changes for complexes $\mathbf{2}$ and $\mathbf{3}$ are presented in Fig. 6.

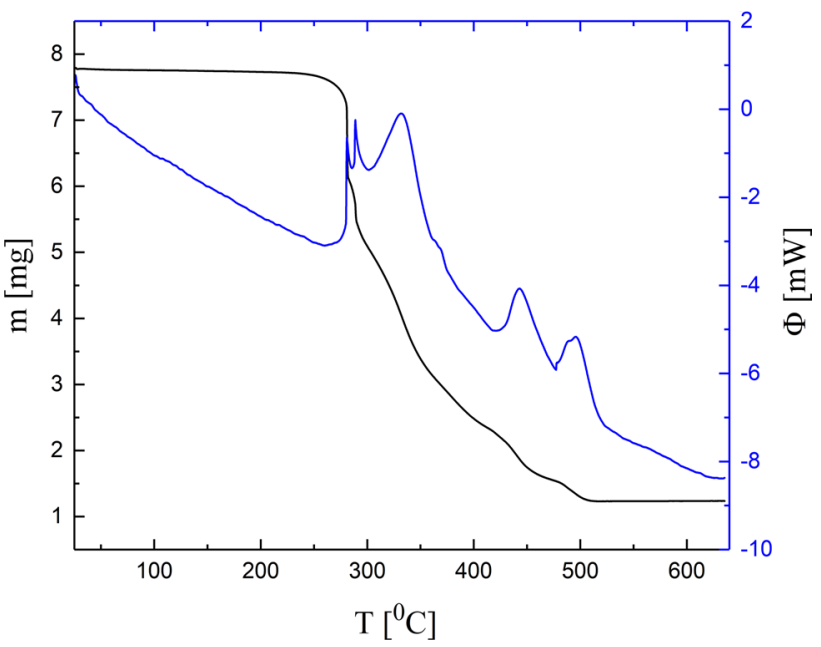

Fig. 9 TG (left axis) and SDTA (right axis) curves for $\mathbf{2}$. Scan speed $10^{\circ} / \mathrm{min}$, air

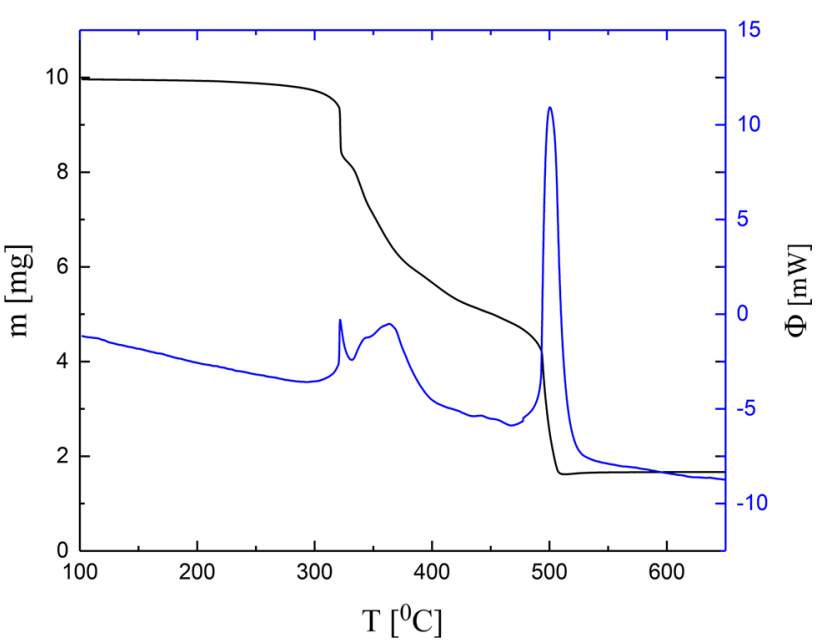

Fig. 10 TG (left axis) and SDTA (right axis) curves for 3. Scan speed $10 \%$ min, air

\section{Cyclic voltammetry}

Cyclic voltammetry measurements were performed in DMSO using $\mathrm{Pt}$ working and counter and $\mathrm{Ag} / \mathrm{AgCl}$ reference electrodes in $0.1 \mathrm{M} \mathrm{Bu}_{4} \mathrm{NPF}_{6}$ as supporting electrolyte. Exemplary voltammograms were selected for oxidovanadium(IV) complexes with phen (Fig. 7) and bpy (Fig. 8) ligands. In general, for oxido complexes of vanadium, irreversible redox processes are observed. Only for complexes with phen or bpy as co-ligand were reversible peaks, attributed to reduction of these ligands, observed in the negative part of the voltammograms. The presence of two reversible processes for 3 (Fig. 7) in this part is a result of the observed instability of $\mathbf{3}$ in DMSO. Thus, we observe "free" and coordinated phen reversible redox processes. As 


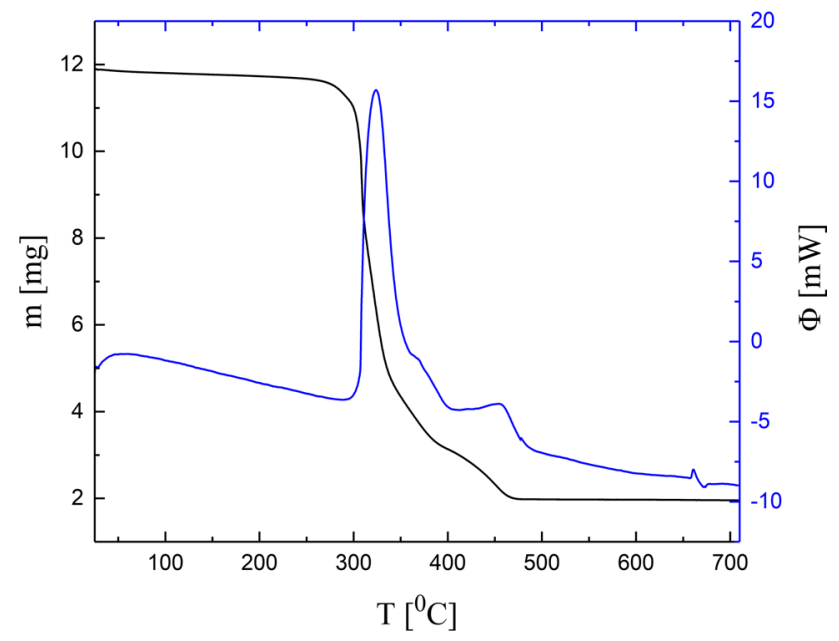

Fig. 11 TG (left axis) and SDTA (right axis) curves for $\mathbf{5}$. Scan speed $10 \%$ min, air

a result of complex stability, Fig. 8 presents the voltammogram of $\mathbf{5}$ which, contrary to $\mathbf{3}$, is stable in DMSO. The presented voltammograms are very similar to those described by us earlier [20-26].

\section{Thermogravimetry}

Thermal decomposition studies were performed in air atmosphere for complexes $\mathbf{2}, \mathbf{3}$, and $\mathbf{5}$. The TG curve together with SDTA results are presented in Figs. 9 10, and 11. The numerical data are presented in Table 3. In general, all the presented complexes are of V(IV) type with co-ligand (bpy or phen). Complicated decomposition processes overlapping with each other were observed. Similarly, as in our previous study, we observed that Schiff base ligand decomposed partly, with hydrazide component released first. A high temperature of solvent molecules release indicates their coordination as ligands. The ligand release in $\mathbf{3}$ is the most typical example. The most volatile hydrazide is released first, while the high-molecular-mass phen ligand is released second. The thermograms of $\mathbf{2}$ and $\mathbf{5}$ with relatively similar composition (the only difference in the benzhydrazide or hydroxybenzhydrazide component of ONO ligand) are very interesting, as they show very different thermal behavior, as presented in Figs. 9 and 11.

As the thermograms were measured in air, the final product is a $\mathrm{V}_{2} \mathrm{O}_{5}$ molecule. The formation of vanadium $(\mathrm{V})$ oxide increases the mass of the final product by $4.46 \%$ for 2 and by $4.31 \%$ for $\mathbf{3}$, and this should be corrected from the final results presented in Table 3, for example from the calculated $88.35 \%$ to $84.05 \%$, which is very close to the experimental value of $83.50 \%$.

\section{Pharmacological activity}

Most of the tested complexes at concentration of $1 \mu \mathrm{M}$ showed similar $(\mathbf{2}, \mathbf{4}, \mathbf{9})$ or weaker $(\mathbf{1 2}, \mathbf{1 3}, \mathbf{1 6})$ activity than BMOV used as a comparator of activity. These results may be explained by structural differences between these complexes. Complex 3 (Table 4) showed significantly lower ability to inhibit all the tested PTPs. This complex is characterized by the greatest differences between the inhibition potency of PTPs associated with antidiabetic activity (PTP1B, SHP1, and SHP2) and the inhibition of CD45 phosphatase. To assess the antidiabetic activity of 3, pharmacological activity studies were performed in cell models (Table 5). The antidiabetic activity of this complex has the same cellular profile and activity as BMOV. Radiolabeled glucose uptake by $\mathrm{C} 2 \mathrm{C} 12$ myocytes is $126 \%$ for 3 and 125\% for BMOV. The effect on glucose uptake in 3T3-L1 adipocytes is negligible for both complexes. In addition, both complexes do not show significant changes
Table 3 TG and SDTA data for complexes $\mathbf{2}, \mathbf{3}$, and $\mathbf{5}$

\begin{tabular}{|c|c|c|c|c|c|}
\hline Complex & $m(\mathrm{mg})$ & $T_{\max }, \operatorname{SDTA}\left({ }^{\circ} \mathrm{C}\right)$ & $\Delta m_{\exp }(\%)$ & $\Delta m_{\text {calc }}(\%)$ & Attributed to \\
\hline 2 & $\begin{array}{l}11.0332 \\
10.9660 \\
10.6505 \\
7.4592 \\
5.1075 \\
2.1618\end{array}$ & $\begin{array}{l}109 \\
309 \\
441 \\
812\end{array}$ & $\begin{array}{l}0.61 \\
3.47 \\
32.39 \\
53.71 \\
80.41\end{array}$ & $\begin{array}{l}4.27 \\
34.98 \\
60.27 \\
87.96\end{array}$ & $\begin{array}{l}0.5 \mathrm{EtOH} \\
\text { bpy, } 0.5 \mathrm{EtOH}, 0.5 \mathrm{H}_{2} \mathrm{O} \\
\text { bpy, } 0.5 \mathrm{EtOH}, \text { hydrazide } \\
\text { bpy, } 0.5 \mathrm{EtOH}, \mathrm{L}\end{array}$ \\
\hline 3 & $\begin{array}{l}10.1038 \\
9.9643 \\
8.1914 \\
4.8075 \\
1.6667\end{array}$ & $\begin{array}{l}321.5 \text { (exo) } \\
345 \text { (exo) } \\
364 \text { (exo) } \\
501 \text { (ехо) }\end{array}$ & $\begin{array}{l}1.38 \\
18.93 \\
52.42 \\
83.50\end{array}$ & $\begin{array}{l}24.83 \\
57.16 \\
88.35\end{array}$ & $\begin{array}{l}\text { hydrazide } \\
\text { phen, hydrazide } \\
1.5 \mathrm{H}_{2} \mathrm{O} \text {, phen, } \mathrm{L}\end{array}$ \\
\hline 5 & $\begin{array}{l}11.8891 \\
11.81331 \\
1.9695\end{array}$ & $\begin{array}{l}324 \text { (exo) } \\
453 \text { (exo) }\end{array}$ & $\begin{array}{l}0.64 \\
83.43\end{array}$ & 87.39 & $\mathrm{H}_{2} \mathrm{O}$, bpy, $\mathrm{L}$ \\
\hline
\end{tabular}

Scan speed $10 \% \mathrm{~min}$ 
Table 4 Inhibition (\%) of human tyrosine phosphatases

\begin{tabular}{llllll}
\hline Complex & $\begin{array}{l}\text { PTB1B } \\
1 \mu \mathrm{M}\end{array}$ & LAR & SHP1 & SHP2 & $\begin{array}{l}\text { CD45 } \\
\text { NOSO }\end{array}$ \\
\cline { 2 - 5 } & 62 & 44 & 70 & 77 & \\
\hline BMOV $^{2}$ & $\mathbf{7 7}$ & 58 & $\mathbf{7 6}$ & $\mathbf{8 2}$ & 40 \\
$\mathbf{2}$ & $\mathbf{7 4}$ & $\mathbf{7 7}$ & $\mathbf{7 8}$ & $\mathbf{8 3}$ & 53 \\
$\mathbf{3}$ & $\mathbf{2 4}$ & $\mathbf{9}$ & $\mathbf{4 6}$ & $\mathbf{5 6}$ & $\mathbf{5}$ \\
$\mathbf{4}$ & $\mathbf{7 5}$ & $\mathbf{7 5}$ & $\mathbf{7 7}$ & $\mathbf{8 3}$ & 42 \\
$\mathbf{5}$ & 58 & 64 & $\mathbf{7 5}$ & $\mathbf{8 2}$ & $\mathbf{3 3}$ \\
$\mathbf{9}$ & $\mathbf{7 3}$ & 62 & $\mathbf{7 5}$ & $\mathbf{8 1}$ & 46 \\
$\mathbf{1 2}$ & 52 & $\mathbf{3 5}$ & 67 & 74 & 29 \\
$\mathbf{1 3}$ & 54 & 46 & 68 & 71 & $\mathbf{1 6}$ \\
$\mathbf{1 6}$ & 50 & $\mathbf{3 3}$ & 68 & 73 & 38 \\
\hline
\end{tabular}

Numerical results expressed as percent inhibition of untreated control (enzyme with solvent only). The highest values are indicated in bold, and the lowest in bold italics. The standard deviation did not exceed $3 \%$ in all cases

Table 5 Pharmacological activity test results for $\mathbf{3}$ and BMOV at concentration of $50 \mu \mathrm{M}$

\begin{tabular}{lrr}
\hline & BMOV & \multicolumn{1}{l}{$\mathbf{3}$} \\
\hline Radiolabeled glucose uptake C2C12 & $125 \pm 6$ & $126 \pm 8$ \\
Radiolabeled glucose uptake 3T3-L1 & $109 \pm 12$ & $103 \pm 6$ \\
Viability HepG2 & $106 \pm 10$ & $106 \pm 15$ \\
Viability C2C12 & $98 \pm 4$ & $104 \pm 2$ \\
Viability 3T3-L1 & $117 \pm 2$ & $107 \pm 8$ \\
Cytotoxicity HepG2 & $100 \pm 1$ & $96 \pm 2$ \\
\hline
\end{tabular}

The results are given as percent of control (solvent only) \pm standard deviation (SD)

in myocyte and adipocyte viability and are not cytotoxic to HepG2 hepatocytes.

The studies of the pharmacological activity of the vanadium complexes showed that they are characterized by diverse activity in inhibiting tyrosine phosphatases. The degree of inhibition of tyrosine phosphatases described as the primary mechanism of action of vanadium compounds need not be crucial for antidiabetic activity such as PTP1B, SHP1, or SHP2. Due to the above, vanadium compound activity studies should include tests in cell models, not just using isolated enzymes.

\section{Conclusions}

The results presented herein indicate that the type of tridentate Schiff base ligand has a huge influence on the biological activity of the studied complexes. In previous publications, we presented a series of Schiff base ONO ligands with the same set of hydrazide component of ligands, with one or two selected aldehydes. Very different biological activity was observed with various hydrazides, but compared with data from other publications, it is obvious that the type of aldehyde used in the synthesis is of similar importance. Here, we present 24 new complexes synthesized with three different aldehydes. In most cases, we used phen as co-ligand to stabilize the oxidation state of V(IV), and here we use also the more flexible bpy coligand, with good stabilization effect. In general, however, more flexible ligand complexes were found to be less stable in solutions. Comparing the biological activity of complexes $\mathbf{2}$ and $\mathbf{3}$, the only difference is the co-ligand used (bpy or phen). It was found that complex $\mathbf{3}$ with the more rigid phen ligand shows similar activity to $\mathrm{BMOV}$, while complex 2 with flexible bpy shows greater activity. In contrast, ca. $32 \%$ of complex 2 remains in DMSO- $\mathrm{H}_{2} \mathrm{O}$ mixture at $60 \mathrm{~min}$ after solution preparation, while complex 3 decomposes almost completely after this time. Similarly, at $\mathrm{pH} 2$, complex $\mathbf{2}$ is slightly more stable than $\mathbf{3}$. All this may indicate that the biological activity of $\mathbf{3}$ is similar to that of BMOV, as both types of complex decompose after injection into simple $\mathrm{VO}^{2+}$ ions. Complex 2 , being more stable, is more effective due to its only partial decomposition, supporting the observed fact that the type of L ligand influences the biological activity and in some cases V(IV) complexes with ONO ligands are threefold more effective than BMOV and human insulin [23].

Acknowledgments This work was partly financed by the European Regional Development Fund under the Innovative Economy Programme 2007-2013 (WND POIG.01.03.01-174/09).

Open Access This article is licensed under a Creative Commons Attribution 4.0 International License, which permits use, sharing, adaptation, distribution, and reproduction in any medium or format, as long as you give appropriate credit to the original author(s) and the source, provide a link to the Creative Commons license, and indicate if changes were made. The images or other third-party material in this article are included in the article's Creative Commons license, unless indicated otherwise in a credit line to the material. If material is not included in the article's Creative Commons license and your intended use is not permitted by statutory regulation or exceeds the permitted use, you will need to obtain permission directly from the copyright holder. To view a copy of this license, visit http://creativecommons.org/licenses/by/4.0/.

\section{References}

1. Tuenter G, Van Leeuwen WF, Snepvangers L (1986) Ind Eng Chem Prod Res Dev 25:633-636

2. Bröer S, Hammer T (2000) Appl Catal B 28:101-111

3. Kamata H, Ohara H, Takahashi K, Yukimura A, Seo Y (2001) Catal Lett 73:79-83

4. McNeill JH, Yuen VG, Dai S, Orvig C (1995) Increased potency of vanadium using organic ligands. In: Srivastava AK, Chiasson 
J-L (eds) Vanadium compounds: biochemical and therapeutic applications. Springer, Boston, pp 175-180

5. Yuen VG, McNeill JH, Orvig C (1995) Can J Physiol Pharmacol 73:55-64

6. Yuen VG, Orvig C, McNeill JH (1993) Can J Physiol Pharmacol 71:263-269

7. Yuen VG, Vera E, Battell ML, Li WM, McNeill JH (1999) Diabetes Res Clin Pract 43:9-19

8. Orvig C, Caravan P, Gelmini L, Glover N, Herring FG, Li H, McNeill JH, Rettig SJ, Setyawati IA, Shuter E, Sun Y, Tracey AS, Yuen VG (1995) J Am Chem Soc 117:12759-12770

9. Ebrahimipour SY, Sheikhshoaie I, Kautz AC, Ameri M, PasbanAliabadi H, Rudbari HA, Bruno G, Janiak C (2015) Polyhedron 93:99-105

10. D'Cruz OJ, Uckun FM (2002) Expert Opin Investig Drugs 11:1829-1836

11. Crans DC, Henry L, Cardiff G, Posner BI (2019) Met Ions Life Sci 19:203-230

12. Torabi S, Mohammadi M, Shirvani M (2018) Trends Pharm Sci 4:87-94

13. Liu EC, Li W, Cheng XS (2019) Acta Chim Slov 66:971-977

14. Qian HY (2017) Russ J Coord Chem 43:780-786

15. Krishnan N, Konidaris KF, Gasser G, Tonks NK (2018) J Biol Chem 293:1517-1525

16. Koren S, Fantus IG (2007) Best Pract Res Clin Endocrinol Metab 21:621-640

17. Niu X, Xiao R, Wang N, Wang Z, Zhang Y, Xia Q, Yang X (2016) Curr Top Med Chem 16:811-822

18. Rehder D (2013) Dalton Trans 42:11749-11761

19. Scior T, Guevara-Garcia JA, Do QT, Bernard P, Laufer S (2016) Curr Med Chem 23:2874-2891

20. Gryboś R, Szklarzewicz J, Jurowska A, Hodorowicz M (2018) J Mol Struct 1171:880-887

21. Szklarzewicz J, Jurowska A, Hodorowicz M, Gryboś R, Matoga D (2019) J Mol Struct 1180:839-848

22. Szklarzewicz J, Jurowska A, Hodorowicz M, Gryboś R, Kruczała K, Głuch-Lutwin M, Kazek G (2020) J Coord Chem 73:986-1008
23. Szklarzewicz J, Jurowska A, Matoga D, Kruczała K, Kazek G, Mordyl B, Sapa J, Papież M (2020) Polyhedron 185:114589

24. Szklarzewicz J, Jurowska A, Hodorowicz M, Gryboś R (2019a) Sci Tech Innov 4:1-8

25. Szklarzewicz J, Jurowska A, Hodorowicz M, Matoga D, Gryboś R, Filipek B, Sapa J, Głuch-Lutwin M, Mordyl B, Kazek G (2019) Sci Tech Innov 4:9-20

26. Jurowska A, Szklarzewicz J, Hodorowicz M, Gryboś R (2019) Sci Tech Innov 4:21-29

27. Szklarzewicz J, Jurowska A, Hodorowicz M, Gryboś R (2019b) Sci Tech Innov 4:30-36

28. Szklarzewicz J, Jurowska A, Olszewska A, Hodorowicz M, Gryboś R, Kruczała K (2019) Sci Tech Innov 4:37-46

29. Hale AJ, Ter Steege E, den Hertog J (2017) Dev Biol 428:283-292

30. Knobler H, Elson A (2014) J Biomed Res 28:157-168

31. Courtney AH, Shvets AA, Lu W, Griffante G, Mollenauer M, Horkova V, Lo WL, Yu S, Stepanek O, Chakraborty AK, Weiss A (2019) Sci Signal 12:8151-8165

32. Dümmling S, Eichhorn E, Schneider S, Speiser B, Würde M (1996) Curr Sep 5:53-56

33. Pastula C, Johnson I, Beechem JM, Patton WF (2003) Comb Chem High Throughput Screen 6:341-346

34. Rigaku Oxford Diffraction (2015) CrysAlis PRO. Rigaku Oxford Diffraction, Yarnton, England

35. Altomare A, Cascarano G, Giacovazzo C, Guagliardi A, Burla MC, Polidori G, Camalli M (1994) J Appl Cryst 27:435

36. Altomare A, Burla MC, Camalli M, Cascarano G, Giacovazzo C, Guagliardi A, Moliterni AGG, Polidori G, Spagna R (1999) J Appl Cryst 32:115-119

37. Sheldrick GM (2015) Acta Cryst C 71:3

38. Schilt A, Taylor RC (1959) J Inorg Nucl Chem 9:211-221

Publisher's Note Springer Nature remains neutral with regard to jurisdictional claims in published maps and institutional affiliations. 